\title{
Métodos geofísicos en entornos naturales protegidos. Tomografía eléctrica
}

\author{
Félix M. Rubio Sánchez-Aguililla(1), Alejandro Ramiro-Camacho(2) y Pedro Ibarra Torre ${ }^{(1)}$ \\ (1) Instituto Geológico y Minero de España (IGME). C/ La Calera 1. Tres Cantos (Madrid). \\ fm.rubio@igme.es \\ (2) Facultad de Ciencias Geológicas. Universidad Complutense de Madrid. C/ José Antonio Novais, 12 (Madrid).
}

\begin{abstract}
RESUMEN
Existe un marcado interés en la protección de los entornos naturales, con el objetivo de su conservación a largo plazo. En ocasiones el valor patrimonial de estas áreas está asociado a su biodiversidad, debido a que existen ecosistemas restringidos que dependen directamente de ellas. En otros casos puede existir un registro geológico singular imprescindible para comprender determinados procesos que afectaron al planeta, como pueden ser eventos volcánicos o períodos glaciares. Para la protección y conservación de estos espacios naturales es necesario tener un conocimiento de la distribución de los materiales y masas de agua en el subsuelo, conocer los parámetros que dominan el comportamiento del sistema y definir aquellos elementos que requieren especial protección.

En estos entornos protegidos es necesario utilizar métodos de investigación que supongan un impacto mínimo sobre el medio. Por ello, las técnicas geofísicas se presentan como una herramienta complementaria, fiable y de mínimo impacto ambiental, para abordar las investigaciones necesarias en estos espacios singulares.

El Instituto Geológico y Minero de España (IGME) ha llevado a cabo trabajos geofísicos en diversos entornos protegidos de España en el marco de investigaciones para mejorar su conocimiento. En esta comunicación se presenta una revisión de casos de aplicación en los que los métodos geofísicos, y en especial el método de tomografía eléctrica, han destacado por su gran utilidad, siendo este último el eje de los estudios geofísicos efectuados. Con ello se pretende divulgar el papel tan importante que este método geofísico puede desempeñar en el estudio de estos espacios singulares.
\end{abstract}

Palabras clave: Geofísica, Hidrogeología, Lago, Resistividad, Tomografía Eléctrica

\section{Geophysical methods in protected environments. Electrical resistivity tomography}

\begin{abstract}
There is a strong interest in protecting the environment with the aim of its long term preservation. Sometimes the heritage value of these natural areas is related to their biodiversity as there are restricted ecosystems that depend directly on them. In other cases there a singular geological record might exist, essential for the understanding of certain processes affecting the planet, such as volcanic events or glacial periods.

To achieve the protection and conservation of these areas it is necessary to generate knowledge about the distribution of geological materials and groundwater masses, to study the parameters that dominate the behaviour of these systems and then define those elements that require special protection or attention.

In these protected environments, research methods with a minimal environmental impact should be used. Therefore, indirect methods, such as geophysical techniques, are reliable and complementary tools with a minimum environmental impact and are therefore useful for research these unique areas.

The IGME has conducted several geophysical surveys in different protected environments in Spain with the aim of achieving a better understanding, and thus facilitate their preservation and exploitation in a sustainable manner. In this paper we present a review of some case studies where geophysical methods have been used. In all the cases electrical resistivity tomography has been the axis of the geophysical research
\end{abstract}


Rubio Sánchez-Aguililla, F.M., et al., 2017. Métodos geofísicos en entornos naturales... Boletín Geológico y Minero, 128 (1): 171-192

and stands out due to its great effectiveness. The main objective of this communication is to divulgate and increase awareness of the important role that these geophysical methods can play in the sustainable study of these unique places.

Keywords: geophysics, hydrogeology, lake, resistivity, electrical resistivity, tomography

\section{ABRIDGED ENGLISH VERSION}

\section{Introduction}

There is a strong interest in the protection of natural areas, with the aim increasing their long-term conservation. In these protected environments it is necessary to use research methods with a minimum environmental impact. A complementary and reliable tool for the study of these unique areas is the use of geophysical techniques. This paper presents a revision of some case studies carried out in several locations in Spain (Fig. 1): The lagoons of Estaña and the Valley of La Larri, in Huesca; the Fuente de Piedra lagoon in Málaga and the Almonte-Marismas aquifer (Huelva and Sevilla). These locations present either unique hydrogeological, geological conditions or ecological heritage and environmental interest, which have motivated the studies presented here. The common geophysical method used in all of them and the one that has provided the most satisfactory information is electrical resistivity tomography (ERT). In some locations, this method has been used together with other geophysical techniques to generate geological interpretations and model objectives for these investigations. In this paper a basic theory of this method is included where topics such as an outline field measurement device and the main arrays used (Fig. 2), electrode distribution and sequence of measurement (Fig. 3), the concept of pseudosection and resistivity model and their differences (Fig. 4), and the main characteristics and comparisons between the various most used arrays are discussed.

\section{Case Studies}

\section{The lagoons of Estaña (Huesca).}

The lagoons of Estaña are located in the province of Huesca (Fig. 1), and are included in the European ecological network Natura 2000 as a Site of Community Importance. The lagoons of Estaña, in the surroundings of the syncline of Estopiñán (Pérez-Bielsa, 2013), are a highly singular karstic complex in the peninsular context with great geological value (Figs. 5a and 5b). The aim of this study was to obtain a reliable geological and hydrogeological model for an intricate lagoon system.

Various geophysical methods were used. Each method separately has helped to establish a geophysical record that was correlated with the available geological and hydrogeological data; they have provided an approach to the hydrogeological model proposed for the lagoons of Estaña. Magnetic resonance soundings (MRS) were to verify the existence of water around the lagoons and their possible link with the Cretaceous limestone of the Estopiñán syncline. ERT was used to determine the continuity of shallow geological structures. Different electrical profiles were performed around the lagoons and connecting with the MRS (Fig. 5b). Six wells were also drilled to obtain hydrogeological information, as well as to support the interpretation of the geophysical data. We also carried out logs of natural gamma and formation resistivity, fluid conductivity and temperature, (Plata and Rubio, 2009; Plata et al., 2010).

In combination with MRS, ERT allowed us to determine the lateral and vertical extension of the lagoons of the Estaña aquifer, as well as to distinguish its different hydrogeological units (Figs. 6 to 10). This was an improvement to the knowledge of the hydrogeological functioning of the lagoons, contributing to defining the key factors controlling the system and, therefore, to its conservation.

\section{The Almonte-Marismas (Sevilla and Huelva) aquifer.}

The aquifer limits to the east with the Guadiamar and Guadalquivir rivers; to the north with outcrops of Miocene blue marls, and to the south with the Atlantic Ocean. It extends a few tens of kilometers offshore and is overburdened by recent coastal sands. The National Park of Doñana is located within the aquifer (Fig. 11). Among the objectives of this study was the definition of the best geophysical methods to properly determine the stratigraphic Miocene-Pleistocene-Holocene transition (to determine the transit between the marls-gravels, sands, silts and clays, the loamy silt of top of the Miocene and the sands of the coastal bar). In 
addition, it was relevant to locate the position of the fresh-salt water interphase in the southeast area of the coastal bar. A series of tests of various geophysical methods was carried out in areas where the geological units and the position of the interphase were well known and controlled, in order to be able to define the most suitable one (Table 1 and Fig. 11). The avilability of wells with necessary geophysical logs and detailed geologic and stratigraphic descriptions was taken into account.

In the Almonte-Marismas aquifer, it was possible to set the geometry of the contact between the dunes and the marls from the ERT, and to distinguish several subunits of different resistivity (Figs. 12 and 13). There was a good correlation between electrical and seismic profiles (Fig. 14). The study provided new information about the hydrogeological performance of the system, optimizing the use of water resources in the area and thus favouring the protection of the associated ecosystems.

ERT has therefore proved to be an effective method for determining lithological vertical contacts between units of different resistivity, even under the phreatic level; however it has not been useful under conditions of strong lateral heterogeneities. ERT also provides satisfactory results for characterizing the geometry of the contact in areas where water shows high salinity contrasts. Nevertheless, it is not possible to distinguish between different lithologies since salt water masks the lithological resistivity contrasts. Similarly, it is difficult to distinguish between bodies of water of different salinity when there are strong lithological resistivity contrasts.

\section{The Fuente de Piedra lagoon (Málaga)}

Another of the unique areas studied is the Fuente de Piedra lagoon in the north-west of the Málaga province (Fig. 1). It is a Natural Reserve, and is one of the Spanish wetlands included in the Ramsar Convention. The Fuente de Piedra lagoon and its basin constitute one of the largest systems of brine in Spain. Due to the endorheic nature of the basin, it is possible to find fresh water in limestone formations and zones with high brine concentration and brackish water with different degrees of salinity (Fig. 15).

Reinterpretation of an old vertical electrical sounding (VES) survey and two new ERT and TDEM surveys were carried out in order to get detailed information to develop a hydrogeological model and improve the hydric management (Fig. 16).

The ERT method has allowed the definition of the available brine levels, both laterally and in depth (Fig. 17). This was of great interest for improving the knowledge of the hydrogeological functioning, and to contribute to the preservation of the Fuente de Piedra lagoon and its associated ecosystems. ERT data were successfully complemented by data from vertical electrical soundings (VES) and time domain electromagnetic soundings (TDES), with all of them offering consistent, compatible results, which reinforce the reliability of the applied techniques.

\section{The La Larri paleolake.}

The last case presented corresponds to the La Larri paleolake, in the north of the Huesca province (Fig.1). The Valley of La Larri is located in the Pineta Valley (headwaters of the Cinca river, on the northeastern edge of the Ordesa and Monte Perdido National Park), in the context of a tectonic window (Pérez-Bielsa, 2013) (Fig. 18). In the Valley of La Larri glacio-lacustrine sedimentary deposits related with later glacial phases to the last local maximum glacier (Salazar-Rincón et al., 2013), are of great interest for the study of glacial cycles in the area. The thickness and extent of these deposits were fundamental concerns in this case.

Two ERT profiles (Fig. 18) were acquired and analyzed together with the information collected from the wells, providing deeper research and continuous lateral information. The results (Figs. 19 and 20) allowed the identification of the different sedimentary units, and the estimation of the thickness of the glacio-lacustrine deposits and the improvement of the understanding of the glacial cycles without the need for new wells.

\section{Conclusions}

As shown in the examples, the application of ERT, alone or together with other methods, provides satisfactory results in environments where great contrasts of resistivity are expected, thus allowing the identification of geological and hydrogeological key units. ERT is a low-cost method, of rapid implementation and low impact on the ground, which reduces the need for wells. These features allow the investigation of the subsurface in areas with high environmental or heritage importance, offering new possibilities for improving knowledge about these environments and thus contributing to their conservation and sustainable use in the future. 
Rubio Sánchez-Aguililla, F.M., et al., 2017. Métodos geofísicos en entornos naturales... Boletín Geológico y Minero, 128 (1): $171-192$

\section{Introducción}

En general, los métodos geofísicos se apoyan en la medición de uno o más parámetros físicos del terreno que posteriormente son interpretados dentro de su contexto geológico para generar un modelo del subsuelo. Cada método geofísico es sensible a una variable física concreta, como la densidad (método gravimétrico), la resistividad (métodos eléctricos y electromagnéticos) o la velocidad de tránsito de las ondas a través del medio (métodos sísmicos). Para abordar el estudio de un sistema hidrogeológico resultan especialmente atractivos los métodos eléctricos y electromagnéticos, debido a que la presencia de agua condiciona la resistividad de la formación en la que se encuentra. De entre los métodos eléctricos, uno de los más extensamente utilizados es el de tomografía eléctrica de resistividad o perfilaje eléctrico, que proporciona secciones de distribución de resistividad en el subsuelo.

En esta comunicación se presentan varios casos de estudio llevados a cabo en las lagunas de Estaña y el valle de La Larri (Huesca), la laguna de Fuente de Piedra (Málaga) y en el acuífero Almonte-Marismas (Huelva y Sevilla). En estas localizaciones (Figura 1) existen singulares condiciones geológicas, hidrogeológicas o ecológicas de elevado interés patrimonial y ambiental, que han motivado las investigaciones aquí presentadas. El método geofísico aplicado en común a todas ellas y que ha proporcionado la información más satisfactoria es la tomografía eléctrica, que se ha utilizado conjuntamente con otros métodos geofísicos para generar las interpretaciones y modelos geológicos objeto de estas investigaciones.

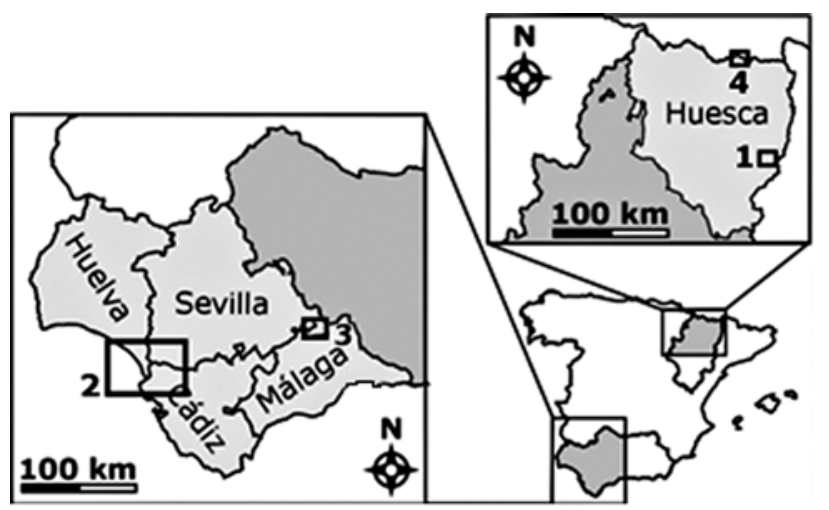

Figura 1. Mapa de localización de las zonas estudiadas. 1: Lagunas de Estaña. 2: Acuífero Almonte-Marismas. 3: Laguna de Fuente de Piedra. 4: Valle de La Larri.

Figure 1. Location map of the studied areas. 1: Lagoons of Estaña. 2: Almonte-Marismas aquifer. 3: Lagoon of Fuente de Piedra. 4: La Larri Valley.
El equipo de tomografía eléctrica empleado en todos los casos ha sido el SAS4000 de la firma sueca ABEM, utilizando el sistema multielectródico Lund Imaging System (ABEM, 2010). En este sistema es posible implantar hasta 60 electrodos, habiéndose utilizado fundamentalmente los dispositivos Wenner y Wenner-Schlumberger. La inversión de los datos de campo y la obtención de los perfiles de resistividad se ha llevado a cabo con el programa RES2DINV de M.H. Loke (Geotomo, 2015)

\section{Método de tomografía eléctrica}

Para obtener resultados satisfactorios en el empleo de las técnicas geofísicas es necesario conocer los fundamentos del método y del procedimiento de inversión de datos que se está utilizando, e interpretar los resultados aplicando un criterio geológico que permita distinguir entre las soluciones geológicamente coherentes y aquellas que no lo son. Los métodos eléctricos, y en concreto la tomografía eléctrica, pueden utilizarse en todos aquellos casos en los que exista un contraste de resistividades entre el objeto de estudio y sus alrededores, lo que permite (entre otras soluciones) estimar la profundidad de un acuífero, localizar zonas fracturadas, o distinguir entre unidades litológicas. La resistividad de las rocas del subsuelo está relacionada con diversos parámetros: los minerales contenidos, la porosidad, el fluido que rellena los poros, el grado de saturación de agua de la roca, la salinidad del fluido, la temperatura, etc. Ejemplos de estudios previos en los que se aplicó este método en estudios hidrogeológicos se pueden encontrar en Ball et al. (2010), Ratnakumari et al. (2012), Galazoulas et al. (2015); en casos de vertederos y detección de cavidades en Meads et al. (2003), Chamberst et al. (2006), Martínez-Pagan et al. (2013) y Martínez-Moreno et al. (2013); en zonas kársticas en Zhou et al. (2002). Además, el casi inexistente impacto que esta técnica produce sobre el terreno hace de ella una opción muy recomendable para investigar el subsuelo de zonas protegidas con fines científicos o medioambientales.

El dispositivo básico de medida (parte superior de la Figura 2) se compone de dos electrodos a través de los cuales se inyecta una corriente de intensidad I al terreno ( $\mathrm{C} 1$ y $\mathrm{C} 2$ en la figura), y dos electrodos en los que se mide la caída de potencial V (P1 y P2 en la figura). A partir de la intensidad de corriente I inyectada al terreno y el voltaje medido $\mathrm{V}$, se calcula un valor de resistividad aparente mediante la expresión $\rho \mathrm{a}=\mathrm{k}$ $\mathrm{V} / \mathrm{I}$, donde $\mathrm{k}$ es un factor geométrico que depende de la disposición y distancia entre los electrodos. La 
medida de resistividad se asigna en el centro del dispositivo, a una profundidad que dependerá del tipo de dispositivo electródico utilizado (Loke, 1999). En la parte inferior de la Figura 2 se muestran los dispositivos más comunes junto con su factor geométrico. Los valores de resistividad calculados mediante esta expresión corresponden a la resistividad que mediría el mismo dispositivo electródico en un terreno homogéneo de resistencia equivalente. Para obtener la resistividad real del terreno a partir de la resistividad aparente es necesario invertir los datos de campo. En los métodos de inversión estándar 1D se supone que el subsuelo está formado por un modelo de capas horizontales en las que la resistividad es constante y solo varía de una capa a otra en profundidad; es el modelo utilizado por los Sondeos Eléctricos Verticales (SEV). La tomografía eléctrica permite utilizar modelos del subsuelo en 2D, donde la resistividad varía no solo con la profundidad sino que también lo hace lateralmente; si bien se asume que la resistividad es constante en dirección perpendicular a la línea de medida (sección o corte del terreno). El uso de tomografía eléctrica, permite por lo tanto estudiar áreas con una geología moderadamente compleja (Griffiths and Barker, 1993).

En estas campañas se trata de ir muestreando

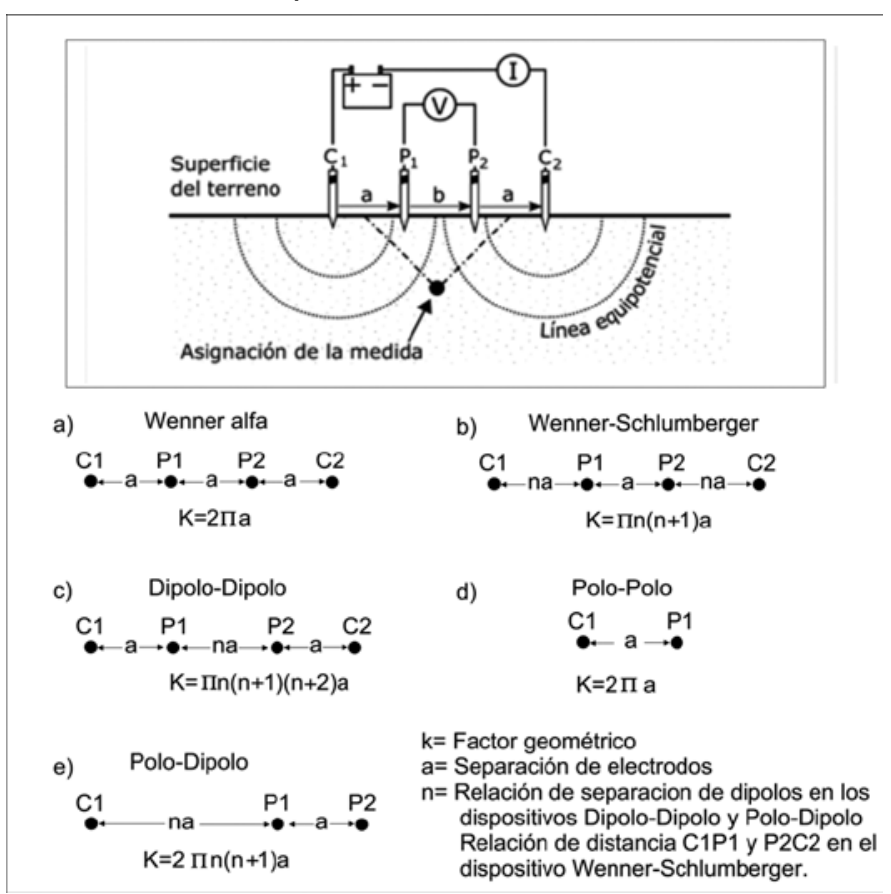

Figura 2. Superior: esquema del dispositivo básico de medición en campo. I: Amperímetro. V: Voltímetro. Inferior (a-d): dispositivos comunes utilizados en campañas eléctricas y sus factores geométricos. Adaptado de Loke (1999).

Figure 2. Top: Basic outline of field measurement device. l: Ammeter. V: Voltmeter. Bottom (a-d): Common electrical devices used in surveys and their geometric factors. Adapted from Loke (1999). la resistividad del subsuelo a diferentes niveles, en puntos cuya equidistancia depende del dispositivo electródico utilizado. Para llevarlas a cabo se utiliza un gran número de electrodos conectados mediante un cable multiconductor (cable con varios hilos conductores). En la Figura 3 se muestra un esquema típico de la forma de llevar a cabo las mediciones en $2 \mathrm{D}$, con los electrodos dispuestos en un perfil según una línea recta.

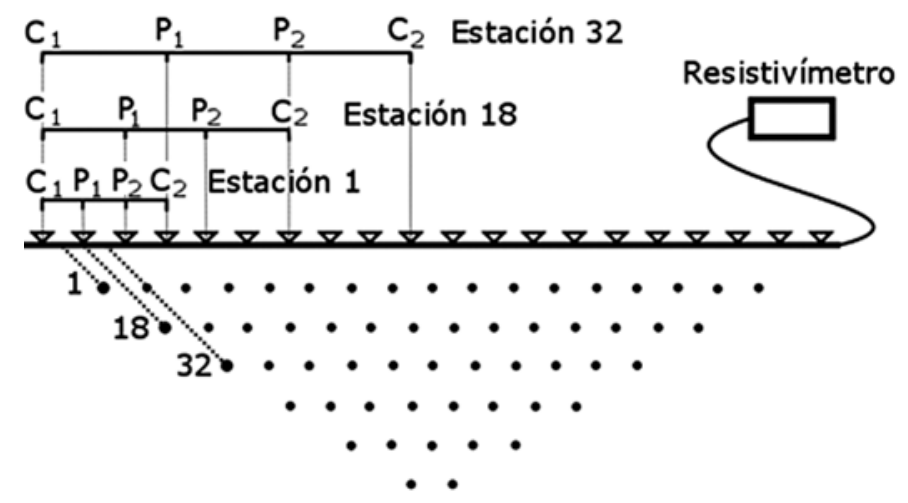

Figura 3. Disposición de electrodos y secuencia de medición para construir una seudosección en una campaña eléctrica 2D. Adaptado de Loke (1999).

Figure 3. Electrode distribution and measurement sequence to build a pseudosection for a $2 D$ electrical survey. Adapted from Loke (1999).

Para representar los datos de la campaña 2D, se utiliza normalmente la seudosección. La localización horizontal de un dato se establece en el punto medio del juego de electrodos utilizados para hacer la medida. La localización vertical del dato se sitúa a una profundidad proporcional a la separación de los electrodos de emisión de corriente. Otro método consiste en situar la posición vertical del dato en la profundidad media de investigación o seudoprofundidad del dispositivo empleado, valor basado en los valores de sensibilidad para un semiespacio homogéneo (Edwards, 1977). Puesto que este último tiene una base matemática, es el que normalmente se emplea. No obstante conviene enfatizar que la seudosección es simplemente una manera de representación que proporciona una imagen aproximada de la distribución de resistividad del subsuelo, que puede estar distorsionada debido a que las isolíneas dependen del tipo de dispositivo empleado así como de la verdadera resistividad del subsuelo. La seudosección es útil como un medio de representar la resistividad aparente medida, y sirve como un modelo inicial para 
la interpretación cuantitativa. Sería un error utilizar la seudosección como una representación final de la resistividad real del terreno (Figura 4) (Loke, 1999).

El uso de diferentes dispositivos puede proporcionar para la misma estructura geológica formas diferentes en las seudosecciones. Cada tipo de dispositivo tiene sus ventajas y sus desventajas, entre las características a tener en cuenta a la hora de elegir un dispositivo están: la sensibilidad del dispositivo a cambios laterales y/o verticales del subsuelo, la profundidad de investigación, la cobertura horizontal del dispositivo y la intensidad de la señal y la susceptibilidad al ruido.

Los dispositivos más comúnmente empleados son (Figura 2): Wenner, Wenner-Schlumberger, DipoloDipolo o Polo-Dipolo. El dispositivo Wenner es el más adecuado para investigar estructuras horizontales, es decir, cambios de resistividad en la vertical del centro del dispositivo y resistividad relativamente homogénea en la horizontal. Comparado con otros dispositivos, permite una moderada profundidad de investigación con la mayor intensidad de señal, lo que puede ser un aspecto determinante en áreas de alto ruido de fondo. Una desventaja de este dispositivo es su relativamente pobre cobertura horizontal.

El dispositivo Dipolo-Dipolo es muy sensible a cambios de resistividad de estructuras verticales, y relativamente insensible a cambios de resistividad de estructuras horizontales. Por ello, es muy útil para resolver estructuras como diques y cavidades, pero relativamente pobre para estructuras donde dominen las capas horizontales. Este dispositivo permite una menor profundidad de investigación comparada con el dispositivo Wenner, pero sin embargo presenta una mejor cobertura horizontal. Una desventaja de este dispositivo es la señal tan débil que se recibe para grandes valores de $\mathrm{n}$ (distancia entre dipolos), por lo que el equipo de medida debe de poseer una alta sensibilidad y un buen sistema de rechazo de ruido.

El dispositivo Wenner-Schlumberger es moderadamente sensible a estructuras verticales (sobre todo profundas) y horizontales (para menor profundidad) y podría constituir un compromiso equilibrado entre los dos dispositivos citados anteriormente. La profundidad de investigación de este dispositivo es alrededor de un $10 \%$ mayor que para el dispositivo Wenner y la intensidad de la señal es menor que la del dispositivo Wenner, aunque mayor que la del dispositivo Dipolo-Dipolo. La cobertura horizontal de este dispositivo es mejor que la obtenida con el Wenner pero peor que la que se obtiene con el Dipolo-Dipolo.

A la hora de realizar la inversión de los datos hay que tener en cuenta que la solución no es única: para los mismos datos medidos existen un amplio rango de modelos que pueden dar lugar a los mismos valores calculados de resistividad. En el caso de que las estructuras del subsuelo posean cambios graduales de resistividad el programa de inversión puede utilizar el algoritmo de inversión de smoothness constraint, el cual trata de minimizar el cuadrado de las diferencias entre los valores de resistividad medidos y calculados. En los casos en que las estructuras presenten cambios bruscos de resistividad (estructuras casi homogéneos internamente y con límites netos), el algoritmo de inversión apropiado es el robust

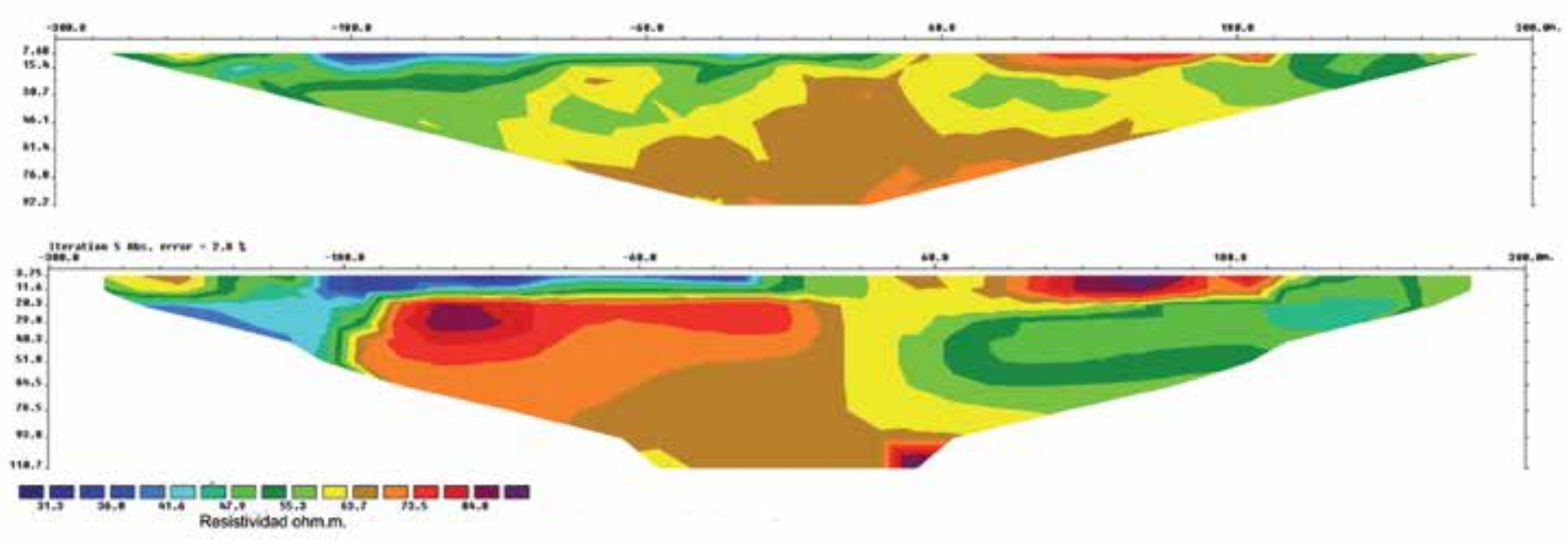

Figura 4. Ejemplo de diferencia entre seudoseccion (parte superior) y modelo de resistividad obtenido tras la inversión de los datos (parte inferior). En este ejemplo la separación entre electrodos es de $15 \mathrm{~m}$, el eje vertical es la profundidad en metros.

Figure 4. Example of difference between pseudosection (top) and resistivity model obtained after inversion of the data (bottom). In the example the unit electrode spacing is $15 \mathrm{~m}$, the vertical left axis is the depth in metres. 
constraint, que trata de minimizar al valor absoluto de las diferencias entre los valores medidos y calculados de resistividad (Loke, 1999).

\section{Ejemplos de aplicación}

A modo de revisión de la aplicación del método de tomografía eléctrica en el estudio de estos espacios protegidos, se exponen trabajos realizados por el área de Geofísica del IGME en cuatro entornos diferentes de la península. La intención es mostrar un resumen de cada uno que permita mostrar la ventaja del empleo de varios métodos geofísicos, su complementariedad, y la aportación de la tomografía eléctrica en particular. En el texto se referencian los informes generados de los diferentes trabajos por si se tiene interés en profundizar en ellos.

\section{Caso 1. Las Lagunas de Estaña (Huesca)}

Las Lagunas de Estaña se sitúan en la provincia de Huesca (Figura 1), y se incluyen en la red ecológica europea Natura 2000 como Lugar de Importancia Comunitaria. Son un conjunto de lagunas naturales de origen kárstico con agua dulce permanente, que se localiza en una cuenca endorreica en la que dominan los carbonatos y las evaporitas.

Las Lagunas de Estaña presentan un elevado valor geológico al ser un sistema kárstico de gran singularidad en el contexto peninsular, encontrándose emplazadas en los alrededores del sinclinal de Estopiñán (Pérez-Bielsa, 2013), un área de gran complejidad geológica. Su valor ecológico es también notable, debido a los bosques de quercíneas y matorrales mediterráneos asociados a estos humedales. De esta singularidad geológica y ecológica surge el interés en mejorar el conocimiento acerca del funcionamiento hidrogeológico de las lagunas.

El humedal de las Lagunas de Estaña se sitúa sobre una franja de materiales triásicos al NE del Sinclinal de Estopiñán, en cuyo núcleo afloran también materiales acuíferos calcáreos del Eoceno y Cretácico Superior (CHE, 2002), separados por materiales poco permeables del Paleoceno en facies Garum. Los materiales del Cretácico Superior constituyen el acuífero principal a nivel regional (Figura 5a).

Se trata de unas lagunas endorreicas de origen kárstico, que se sitúan sobre materiales carbonatados y dolomíticos triásicos de las facies Muschelkalk, que afloran como bloques aislados de extensión hectométrica, y materiales arcillosos y evaporíticos de las facies Keuper con espesores de hasta 250 m (IGME, 1994, 1995, 2007) (Figura 5b). Dentro del Keuper afloran también una serie de masas de ofitas (Lago y Pocovi, 1982).

Los materiales del Sinclinal de Estopiñán se relacionan con los del Triásico por medio de un contacto mecánico, correspondiente probablemente a una falla inversa, que pone en contacto los materiales del Cretácico Superior (de edad SantonienseCampaniense) con los del Keuper y puntualmente también con los del Muschelkalk.

A pesar de tratarse de un área de importancia ambiental reconocida, la hidrogeología y los límites hidrogeológicos no habían sido suficientemente estudiados (Pérez-Bielsa et al., 2012). Por ello se propusieron trabajos geofísicos encaminados a proporcionar información para desarrollar un modelo hidrogeológico que permita determinar las relaciones entre el agua subterránea y las lagunas, y establecer límites hidrogeológicos en su entorno. En particular, se estudió la continuidad en profundidad de los materiales cartografiados (limos con cantos sueltos del Cuaternario, arcillas y yesos del Keuper, y dolomías y calizas del Muschelkalk) así como el contacto entre las calizas del Cretácico Superior (Sinclinal de Estopiñán) y el Keuper (Plata and Rubio, 2009).

Para alcanzar estos objetivos se han empleado diversos métodos geofísicos y este estudio constituye un ejemplo excelente de cómo se complementa la información procedente de diferentes técnicas geofísicas. Cada método por separado ha permitido establecer unos documentos geofísicos, que correlacionados con los datos geológicos e hidrogeológicos disponibles, han proporcionado una aproximación al modelo hidrogeológico que caracteriza a las Lagunas de Estaña.

Los Sondeos de Resonancia Magnética (SRM) constituyen un método capaz de detectar de manera directa la presencia de agua, y se ha empleado para verificar la existencia de agua alrededor de las lagunas y su posible conexión con las calizas cretácicas del sinclinal de Estopiñan. La tomografía eléctrica se ha utilizado para determinar la continuidad de las estructuras geológicas superficiales. Los distintos perfiles eléctricos se han distribuido alrededor de las lagunas y conectando los SRM. Asimismo se han realizado 6 sondeos mecánicos con recuperación de testigo, con profundidades que variaban entre $15 \mathrm{y}$ $144 \mathrm{~m}$ para obtener información de carácter hidrogeológico, así como para apoyar la interpretación de los datos geofísicos. Además, se realizó la testificación geofísica de gamma natural, resistividad de la formación, conductividad y temperatura del fluido. La distribución de estos trabajos se observa en la Figura 5b. Un mayor desarrollo del trabajo de análisis 

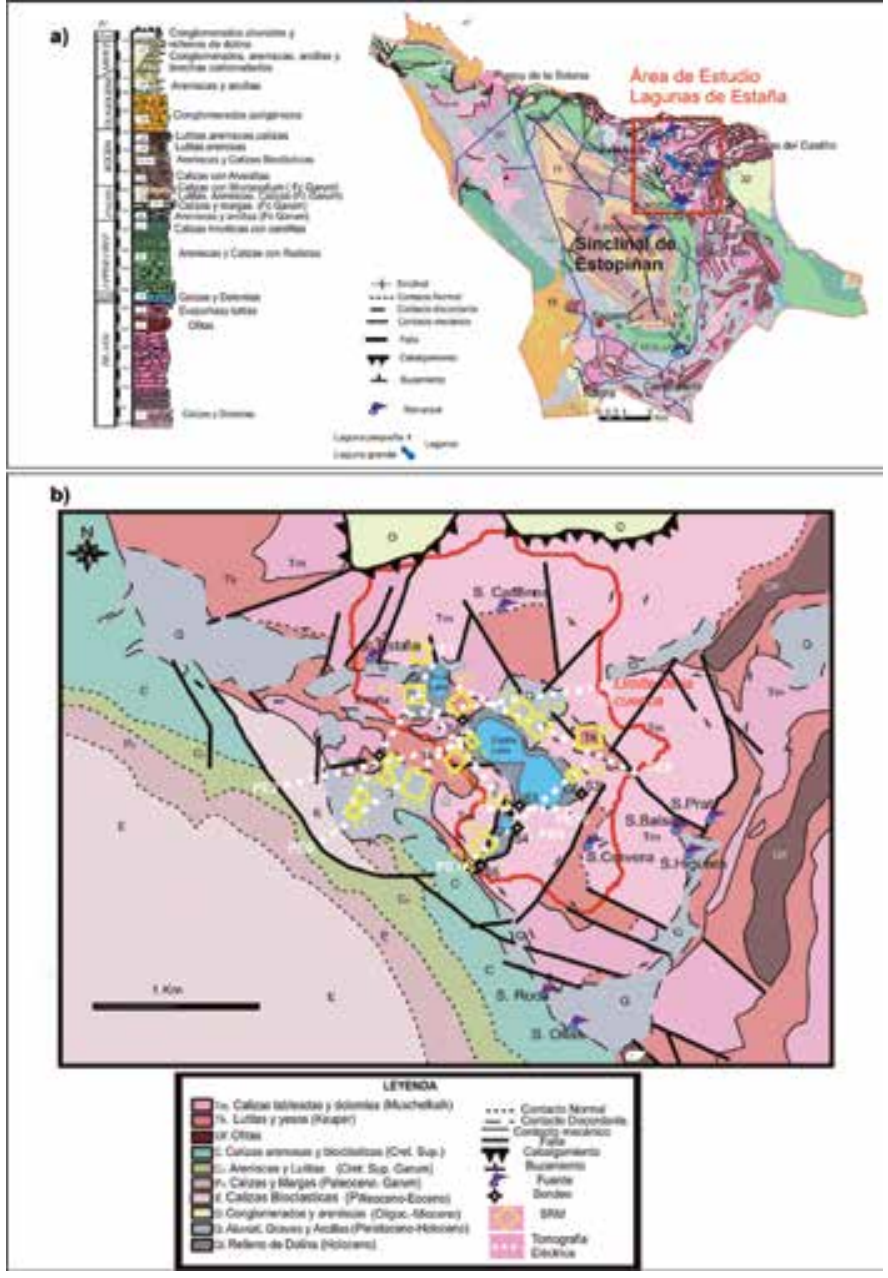

Figura 5. a) Mapa geológico regional de la zona de estudio, donde se indica el sinclinal de Estopiñán. El cuadrado rojo señala la ubicación de las Lagunas de Estaña. b) Mapa geológico de detalle de los alrededores de las lagunas de Estaña. En la figura se muestra la distribución de los trabajos geofísicos realizados en las lagunas de Estaña. (Ambas figuras modificadas de Bielsa et al. 2013).

Figure 5. a) Regional geological map of the study area, Estopiñan syncline is indicated. Red square shows the site of the lagoons of Estaña. b) Detailed geological map in the surroundings of the lagoons of Estaña. Distribution of the geophysical works performed is indicated. (Both figures modified from Bielsa et al. 2013).

efectuado en este Proyecto se encuentra en Plata and Rubio (2009) y Plata et al. (2010).

Se han relacionado los valores de las testificaciones en los sondeos con su columna litológica, y con las interpretaciones de los SRM; también se ha relacionado la resistividad procedente de los perfiles eléctricos con la litología y los valores de las diagrafías (Figura 6 a y b), otra forma de establecer esa relación ha sido verificando los valores de la resistividad superficial de las secciones eléctricas con la cartografía geológica, cuando existe garantía de que todos los electrodos están sobre el mismo tipo de terreno. A partir de estas relaciones se ha establecido un rango de resistividades característico de las diferentes unidades geológicas presentes (Figura 7). Una vez establecida la relación más probable entre la resistividad medida en los perfiles eléctricos y la litología, se ha realizado una interpretación geológica de los perfiles (Figura 8).

Del análisis e interpretación de las secciones se deduce que la distribución de la resistividad no está excesivamente apartada de un modelo 2D para la mayoría de los lugares medidos, lo que permite realizar
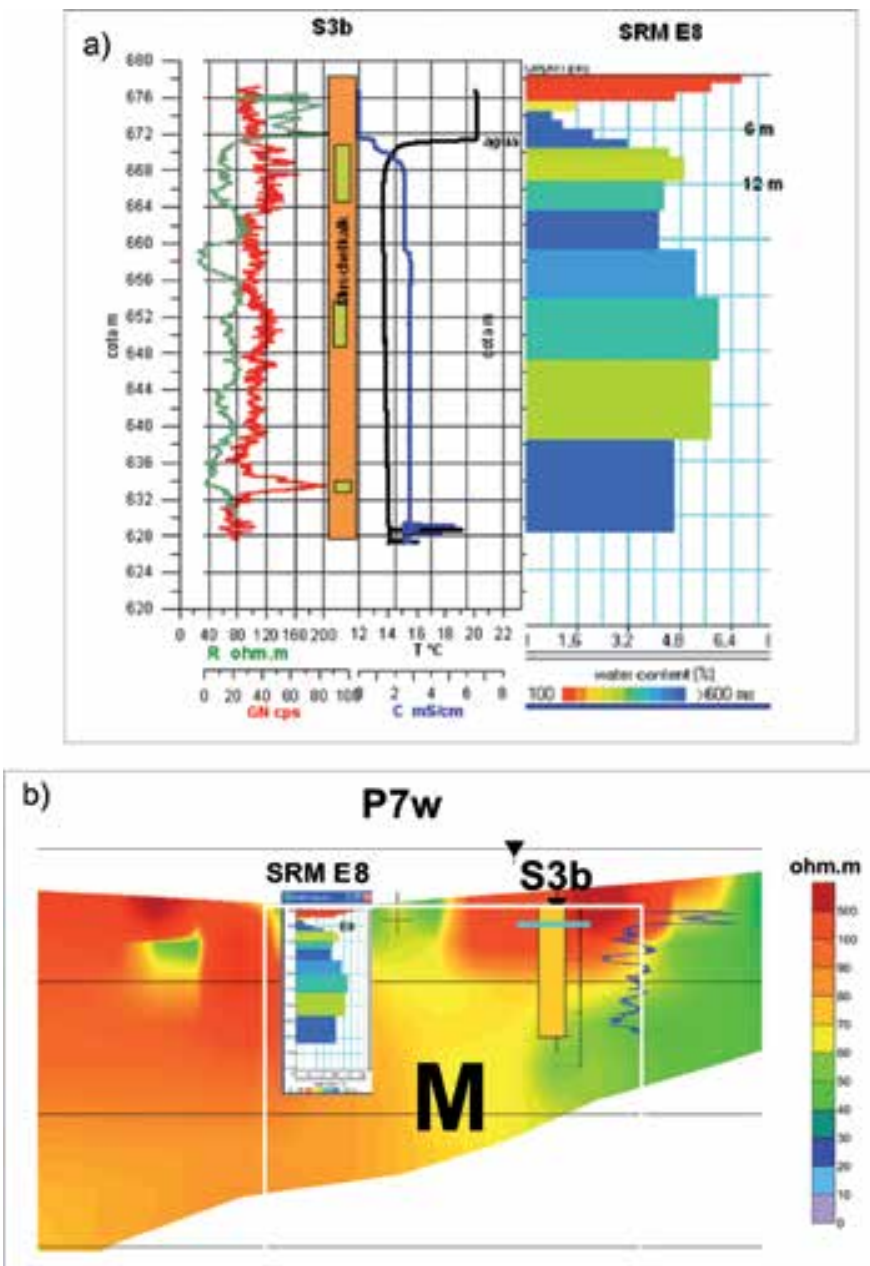

Figura 6. a) Ejemplo de los registros obtenidos en el sondeo $3 b$, junto con la interpretación del SRM E8 realizado en su proximidad. b) Ejemplo de la correlación realizada entre el perfil eléctrico, la interpretación del SRM y la información procedente de las diagrafías medidas en el sondeo mecánico.

Figure 6. a) Example of logs measured in well $S 3 b$, and the interpretation of MRS E8 measured near the well. b) Example of the integration of the logs and SRM interpretation into the electrical profile in order to correlate the resistivity value. 


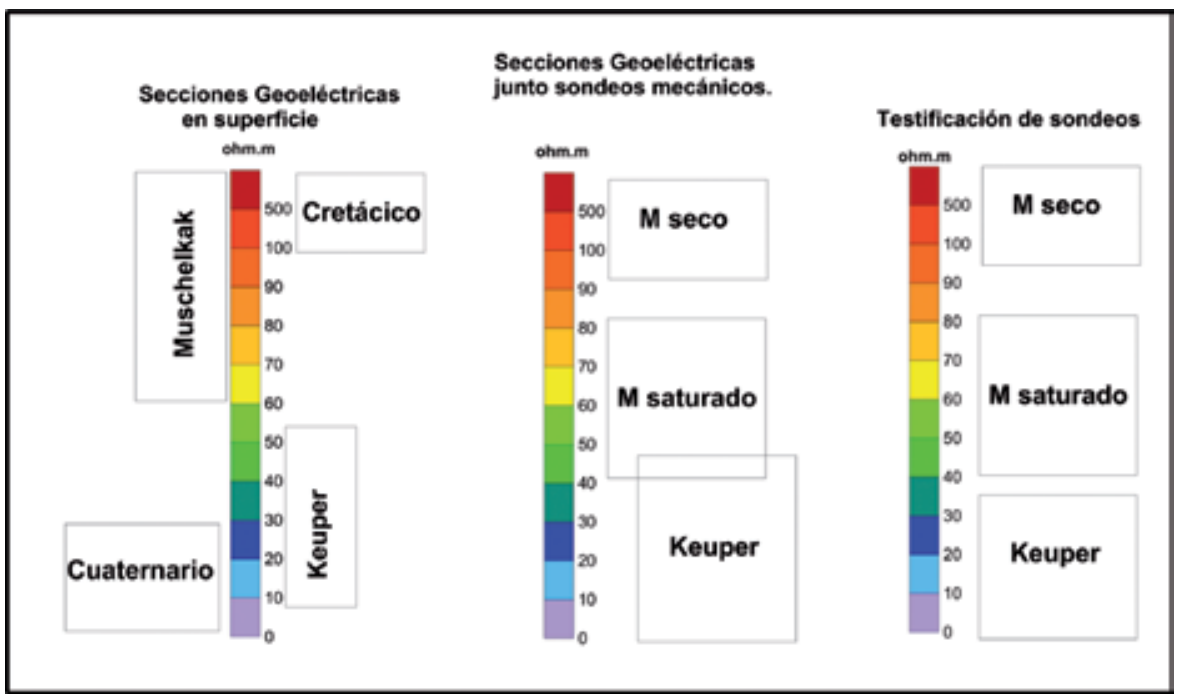

Figura 7. Asignación de resistividad a las unidades geológicas según la información de resistividad superficial (izquierda), según la información junto a los sondeos mecánicos (centro) y la procedente de la testificación de los sondeos mecánicos (derecha).

Figure 7. Resistivity allocation to geological units from surface resistivity information (left), information from nearest wells (center), and from resistivity logs (right).

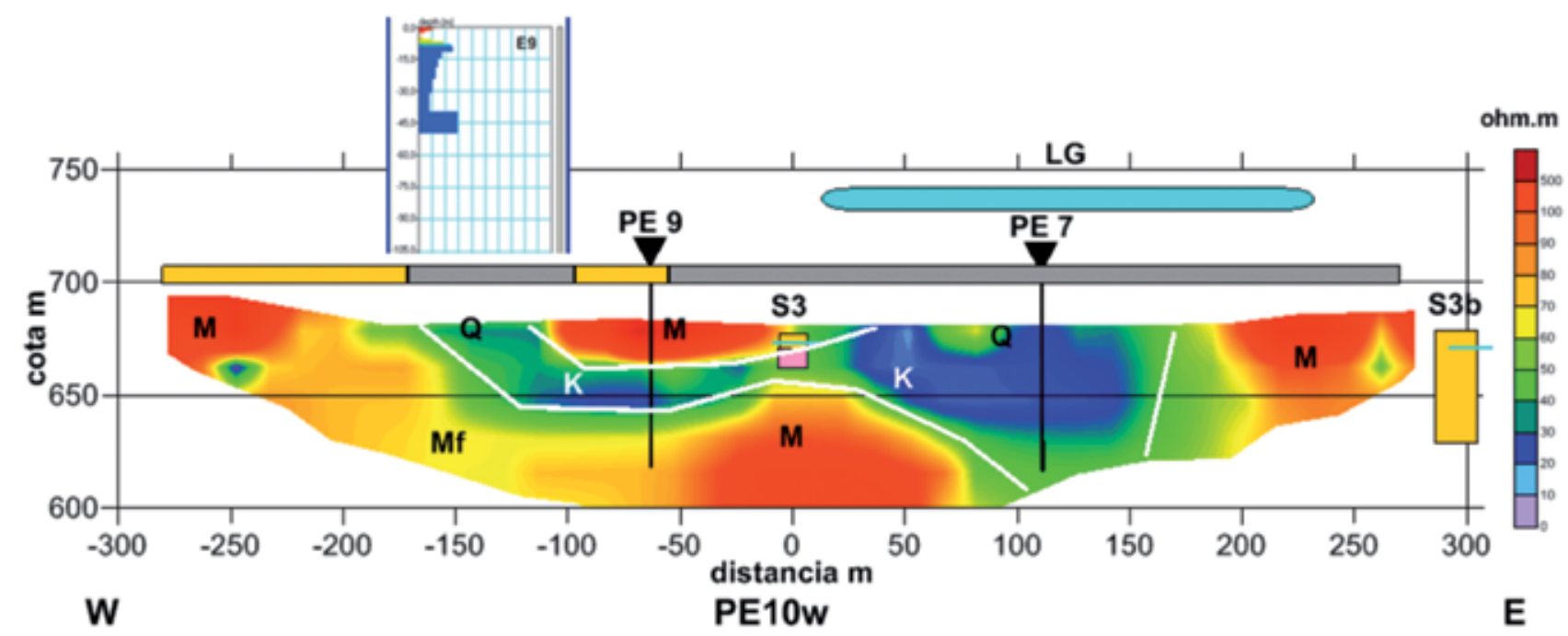
Q: Cuaternario
M : Muschelkalk
Mf : Muschelkalk fracturado
K : Keuper
LG : Laguna grande
S3 y S3b : Sondeos mecánicos S3 yS3b
E9 : SRM E9

Figura 8. Interpretación geológica del perfil 10 en imagen de distribución de resistividad.

Figure 8. Geological interpretation of the profile 10.

una interpolación de la resistividad entre perfiles. Esto permite trazar mapas de distribución de resistividad a diferentes profundidades (Figura 9). Aunque existe cierta incertidumbre de la representatividad de dicha interpolación, ver los detalles de la misma en Plata et al. (2010), lo que los mapas permiten es comparar los valores de resistividad con otros datos que no están medidos exactamente sobre los perfiles eléctricos, como mejor alternativa que proyectar su posición directamente sobre las secciones de resistividad. Estos mapas permiten además comprender mejor la evolución tridimensional de la geología de la zona.

Una vez establecida la correspondencia entre la resistividad y las ubicaciones en las que tanto los sondeos mecánicos como los SRM han detectado la 
presencia de agua, se puede establecer una clasificación en términos hidrogeológicos de la resistividad, como se indica en la Figura 9. Con esta clasificación se han vuelto a trazar las secciones geoeléctricas, incluyendo los SRM que realmente se manifiestan en cada sección.

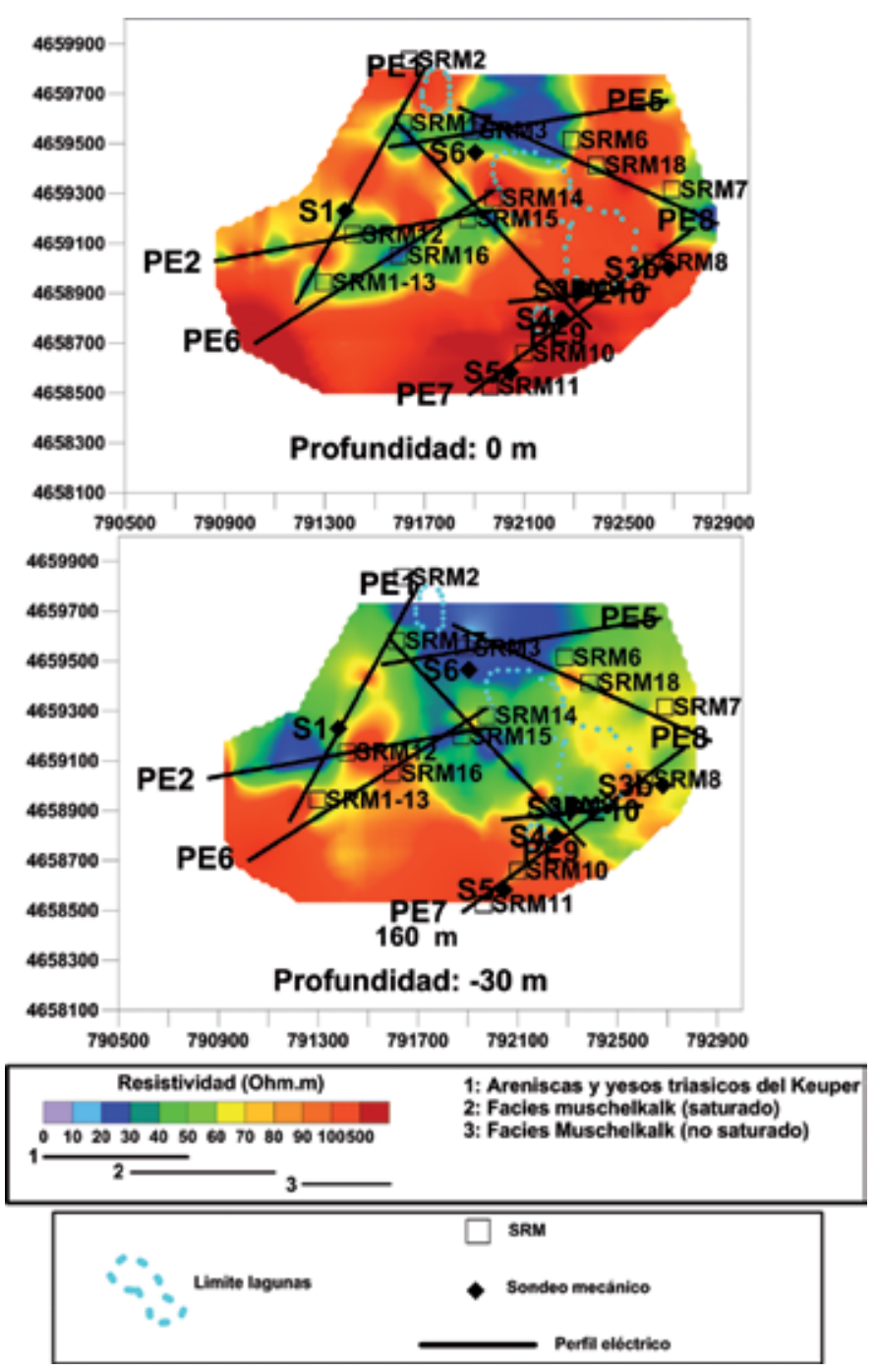

Figura 9. Mapas de distribución de la resistividad a 0 y $30 \mathrm{~m}$ de profundidad. Se indica la posición de los perfiles, cuyos datos utilizados para la construcción del mapa quedan dentro del perímetro de la imagen. Las lagunas están representadas por puntos azules. Se indica también la posición de los SRM (cuadrados) y los sondeos mecánicos $\mathrm{S}$ (rombos). En la parte inferior se muestra la asignación de resistividad en términos hidrogeológicos. (Coordenadas UTM ED50 H30N).

Figure 9. Maps of resistivity distribution at 0 and $30 \mathrm{~m}$ depth. The position of the profiles used to draw the map (data are within the perimeter of the image) is indicated. Lagoons are represented by blue dots. The position of the MRS (squares) and boreholes S (diamonds) is also indicated. Bottom, allocation of resistivity in hydrogeological terms. (Coordinates UTM ED50 H3ON).

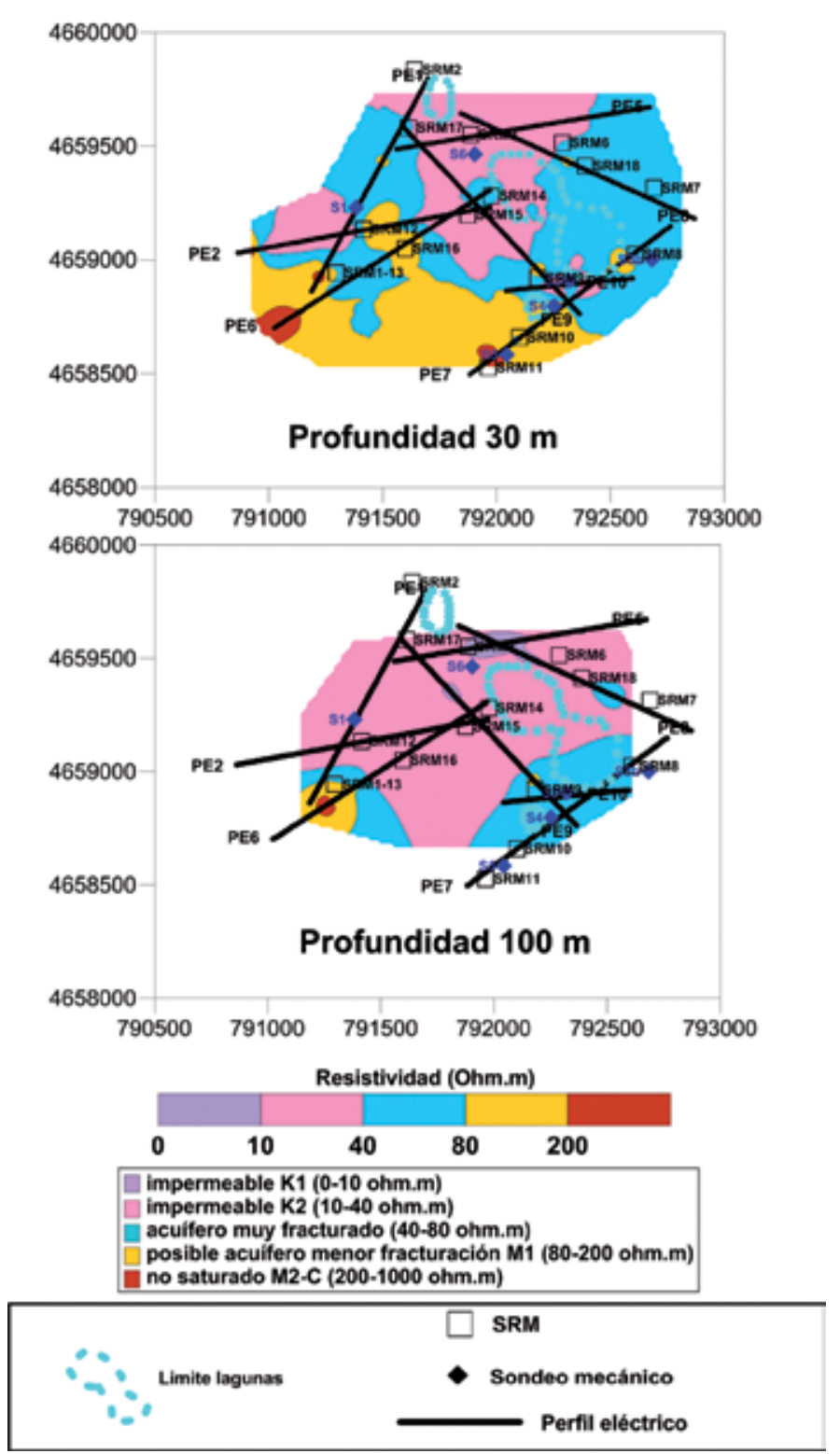

Figura 10. Interpretación de la situación hidrogeológica a 30 y 100 $\mathrm{m}$ de profundidad, de acuerdo a la relación propuesta entre la resistividad, la litología y el contenido en agua (cuadro inferior). (Coordenadas UTM ED50 H30N).

Figure 10. Interpretation of hydrogeological situation at 30 and 100 $m$ deep, according to the proposed relationship between the resistivity, lithology and water content (lower panel). (Coordinates UTM ED50 H30N).

Se han realizado mapas a varias profundidades, que han permitido obtener una visión de la evolución espacial del acuífero, así como de la conexión de las diferentes partes del mismo (Plata et al. 2010), y que como ejemplo en la figura 10 se muestran los correspondientes a 30 y $100 \mathrm{~m}$ de profundidad. La aparente compartimentación estanca de las rocas permeables 
que se observaba en los cortes, toma en su visión en planta un aspecto de mayor continuidad. El acuífero es libre en todo el sector central, y está limitado en sus flancos oriental y meridional por rocas carbonatadas y al norte por el Keuper, aunque quedando parcialmente abierto en los bordes norte y occidental. Los materiales impermeables del Keuper son más abundantes en profundidad, de tal forma que a partir de $30 \mathrm{~m}$ de profundidad su presencia condiciona la existencia de un acuífero oriental, semi-confinado, y otro occidental, libre, comunicados por un canal (Figura 10). Esta situación se mantiene hasta al menos los $100 \mathrm{~m}$ de profundidad, a partir de donde es posible que todo el basamento sea impermeable (Figura 10). En el borde sur de la zona, ocupado por materiales carbonatados no saturados, solo se presenta un cierto grado de fracturación sobre los $30 \mathrm{~m}$ de profundidad.

\section{Caso 2. El acuífero Almonte-Marismas}

La zona propuesta para su estudio se encuentra situada en el sistema acuífero Almonte-Marismas en la depresión del Guadalquivir. El acuífero limita al este con los ríos Guadiamar y Guadalquivir; al norte con los afloramientos de margas azules del Mioceno y al sur con el océano Atlántico, prolongándose mar adentro unas decenas de kilómetros y recubierto por arenas litorales recientes. Ocupa una superficie de $3400 \mathrm{~km}^{2}$, de los cuales unos $1000 \mathrm{~km}^{2}$ corresponden al sector confinado bajo las marismas, y tiene un espesor variable entre unas pocas decenas de metros tierra adentro y más de $200 \mathrm{~m}$ bajo la marisma, cerca de la costa (Plata et al., 2007). En el interior del acuífero se encuentra el Parque Nacional de Doñana (Figura 11).

En conjunto, este sistema se puede considerar compuesto por un acuífero aluvial multicapa de limos, arenas y gravas de origen fluvio-deltaico y marino, que aflora en la mitad occidental y se sitúa en la oriental bajo una gruesa capa de arcillas de baja permeabilidad en las marismas, comportándose en esta zona como un acuífero confinado (Plata et al., 2007). A este acuífero se le superpone otro de arenas de origen eólico que cubre todo el frente costero. La recarga tiene lugar a través de las arenas eólicas y de la parte libre del acuífero detrítico. El flujo subterráneo tiene una dirección general NO-SE, encontrándose el acuífero en carga en situación natural bajo las arcillas de la marisma, jugando dicha formación un papel importante en el funcionamiento hidráulico del sistema (Plata et al., 2007).

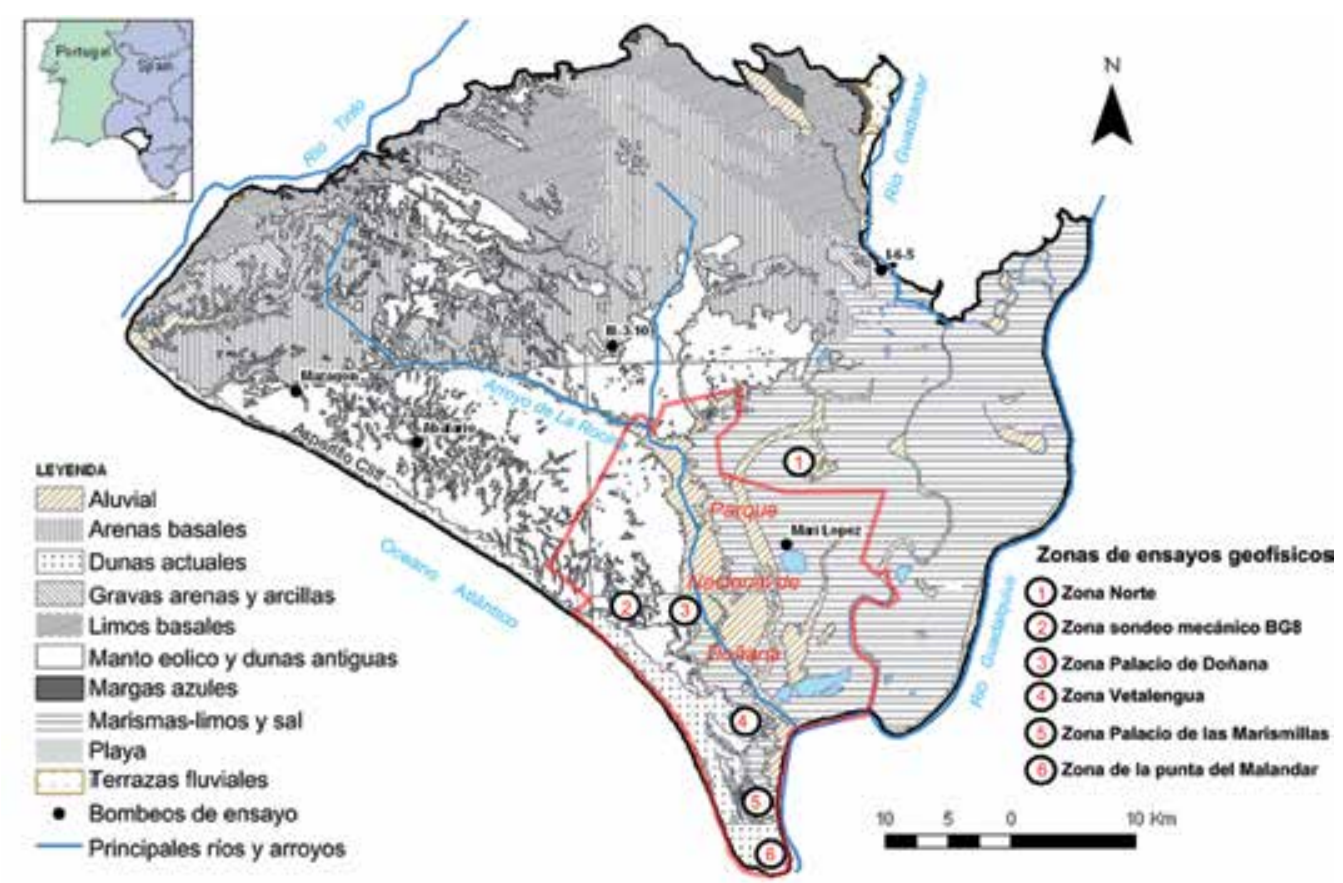

Figura 11. Mapa Geológico del acuífero Almonte-Marismas. Se indican las diferentes zonas de estudio donde se ensayaron los métodos geofísicos de superficie.

Figure 11. Geological map of the Almonte-Marismas aquifer. Estudy areas where essays of surface geophysical methods have been carried out are shown. 
Los trabajos técnicos realizados desde 1964 permiten concluir que la geología, geometría y funcionamiento hidráulico del sistema acuífero AlmonteMarismas pueden considerarse conocidos en sus rasgos generales, y con más detalle solo en los lugares donde se realiza su explotación. Sin embargo, la coexistencia de dos sectores, uno libre, donde tiene lugar la recarga del acuífero y donde se ubican la mayor parte de las explotaciones, y otro semiconfinado bajo la marisma, junto con los diferentes mecanismos existentes en la zona para las relaciones de interfase entre agua dulce y agua salada, confieren al acuífero una especial complejidad, no verificándose siempre en trabajos de detalle las hipótesis establecidas con carácter más general (Plata et al., 2007).

Se planteó necesario mejorar el conocimiento de la geometría y funcionamiento del acuífero, tanto a nivel general como de detalle en determinadas zonas más restringidas. Esto es de gran importancia para compatibilizar la explotación del acuífero con la conservación del medio ambiente, siendo esto último prioritario en un entorno de gran riqueza ecológica. Los trabajos realizados estuvieron encaminados a la mejora de los modelos conceptual y numérico existentes del acuífero.
Entre los objetivos propuestos se encuentra el de definir la mejor metodología geofísica para resolver problemas estratigráficos de determinación del tránsito Mioceno-Pleistoceno-Holoceno (determinar el tránsito entre el relleno pleistoceno-holoceno de la marisma, -gravas, arenas, limos y arcillas-, los limos margosos del techo del Mioceno y las arenas de la barra de costa), así como para situar la posición de la interfase agua dulce-agua salada en la zona suroriental de la barra litoral. Para ello se efectuaron una serie de pruebas de diversos métodos geofísicos en áreas donde las diferentes temáticas propuestas se encuentren bien conocidas y controladas, a fin de poder definir el más adecuado. Se tuvo en cuenta la disponibilidad de sondeos mecánicos con las necesarias testificaciones geofísicas y una descripción geológica y estratigráfica detallada (Plata et al, 2007).

Las zonas de ensayo seleccionadas y los métodos geofísicos de superficie efectuados se muestran en la Tabla 1 y la Figura 11.

En la zona de Vetalengua se realizaron pruebas para determinar el método más adecuado para delimitar la profundidad y forma de la interfase de las aguas salobres bajo la marisma y el manto eólico, así como el contacto dunas-marisma. Se utilizó el

\begin{tabular}{|c|c|c|c|c|c|c|c|}
\hline & \multicolumn{7}{|c|}{ Zonas de realización de las campañas en el Acuifero Almonte-Marismas } \\
\hline & Vetalengua & $\begin{array}{l}\text { Palacio de } \\
\text { Marismillas }\end{array}$ & Malandar & $\begin{array}{c}\text { Junto } \\
\text { sondeo } \\
\text { BG8 }\end{array}$ & $\begin{array}{l}\text { Palacio de } \\
\text { Doñana }\end{array}$ & $\begin{array}{l}\text { Zona Norte } \\
\text { (M-6-5) }\end{array}$ & Santa Olalla \\
\hline $\begin{array}{c}\text { Perfiles de } \\
\text { Sismica de reflexión }\end{array}$ & & $3 \mathrm{~km}$ & & $1 \mathrm{~km}$ & $0,95 \mathrm{~km}$ & $2,8 \mathrm{~km}$ & \\
\hline Ensayos Down Hole & & $190 \mathrm{~m}$ & & & $190 \mathrm{~m}$ & $197 \mathrm{~m}$ & \\
\hline $\begin{array}{c}\text { Sondeo } \\
\text { electromagnético } \\
\text { en el Dominio del } \\
\text { tiempo } \\
\text { (SEDT) } \\
\end{array}$ & 3 & 18 & & & 3 & & 1 \\
\hline Tomografia Eléctrica & $1,3 \mathrm{~km}$ & & & & & & \\
\hline $\begin{array}{c}\text { Sondeos Eléctricos } \\
\text { Verticales } \\
\text { (SEV) } \\
\end{array}$ & 3 & 9 & & 1 & 3 & & 1 \\
\hline Tomografia Eléctrica & $1,5+3,7 \mathrm{~km}$ & $3 \mathrm{~km}$ & $1,5 \mathrm{~km}$ & $1,2 \mathrm{~km}$ & $1,5+5 \mathrm{~km}$ & $3,3 \mathrm{~km}$ & \\
\hline
\end{tabular}

Blanco : Realizados mediante asistencia técnica Gris: Realizados con los equipos del IGME

Tabla1. Tabla resumen de los trabajos de geofísica de superficie llevados a cabo en el Acuífero Almonte-Marismas.

Table 1. Summary of surface geophysical surveys carried out in the Almonte-Marismas aquifer. 
sondeo mecánico S-57 como control para evaluar la efectividad de los métodos geofísicos de superficie (Figura 12a). La testificación litológica del sondeo reveló que en ese punto dominan las arcillas en toda la columna perforada, y la testificación geofísica de la conductividad del fluido indica la existencia de aguas muy saladas en toda la columna (Figura 12b).
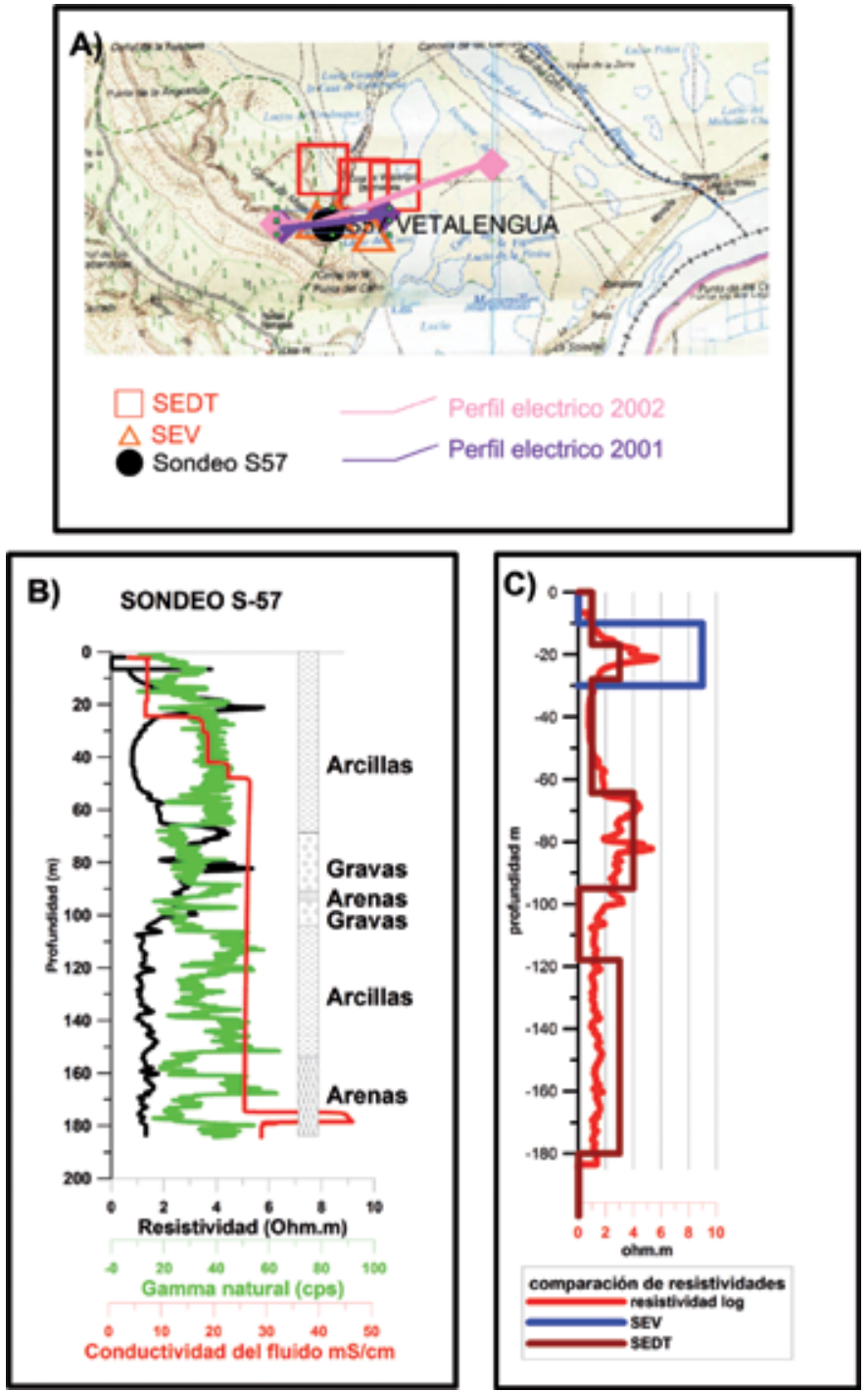

Figura 12. A) Situación de los SEV, SEDT y perfiles eléctricos realizados en Vetalengua junto con la situación del sondeo S57 que se tiene como referencia. B) Registros realizados en el sondeo mecánico junto con su columna litológica. C) Correlación del registro de resistividad en el sondeo con los valores de resistividad de SEVs y SEDTs próximos.

Figure 12. a) Location of the VES, TDEM and ERT carried out in Vetalengua, and the $S 57$ Borehole, taken as reference. B) Logs measured in the $S 57$ well and its lithological column. C) Correlation between the resistivity log and resistivity values from nearest VES and TDEM.
Se han ejecutado perfiles eléctricos, SEV y SEDT próximos al sondeo mecánico. La comparación de la testificación de resistividad del sondeo con los valores de resistividad de los SEV y SEDT muestra una secuencia de resistividades coincidente (Figura 12c).

Se realizaron 2 perfiles eléctricos, uno en el año 2001 y otro al año siguiente, este último más largo y adentrándose más en la marisma (Figura 13). La correlación del sondeo y sus registros con el perfil eléctrico muestra una secuencia similar de resistividades, si bien evidencia las fuertes variaciones laterales existentes, por lo que los datos del sondeo son difícilmente extrapolables a una geometría con continuidad subhorizontal (Figura 13). El contacto dunas-marisma queda claramente identificado mediante tomografía eléctrica, sin embargo, la apreciación del límite del agua dulce-agua salada no es viable, ya que no es posible diferenciar si la variación de resistividad se debe a la salinidad del agua de formación o a un cambio de litología. No se observa una correlación directa entre la variación de resistividad y los distintos contactos litológicos determinados a partir de sondeos cercanos, por lo que la resistividad medida en esta zona del perfil se atribuye a una superposición de ambos efectos.

Las condiciones de medición de estos perfiles (dificultad de buenos contactos eléctricos, gran heterogeneidad de resistividades, etc.), pueden ser la causa de gran parte de las fuertes anomalías puntuales,
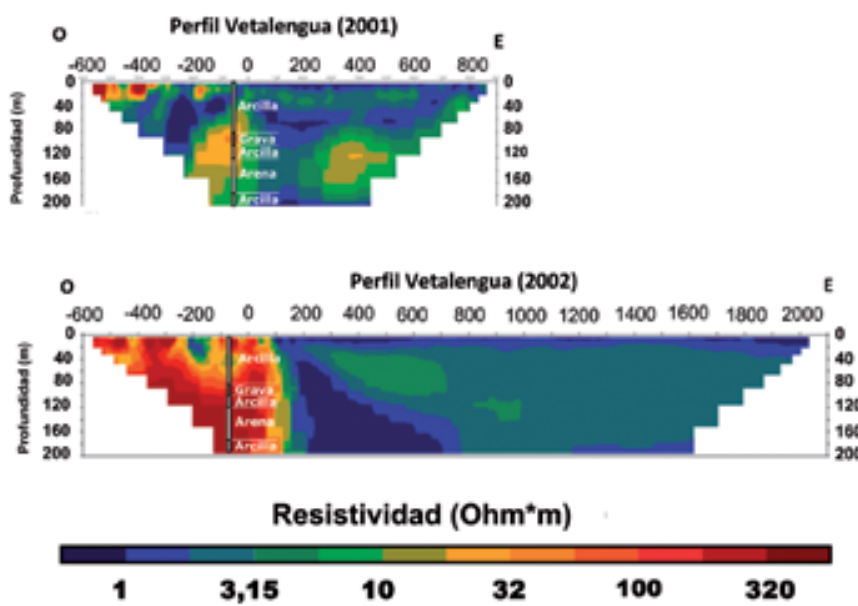

Figura 13. Perfiles eléctricos Wenner medidos en la zona de Vetalengua (Almonte-Marismas). En ambos se indica la situación del sondeo mecánico y su columna litológica. Parte superior: perfil medido en 2001. En la parte inferior se muestra el perfil medido en 2002.

Figure 13. Wenner electrical profiles carried out in the Vetalengua zone. Lithological column of the $S 57$ well are included in both profiles. Upper: profile measured in 2001. Bottom: profile measured in 2002. 


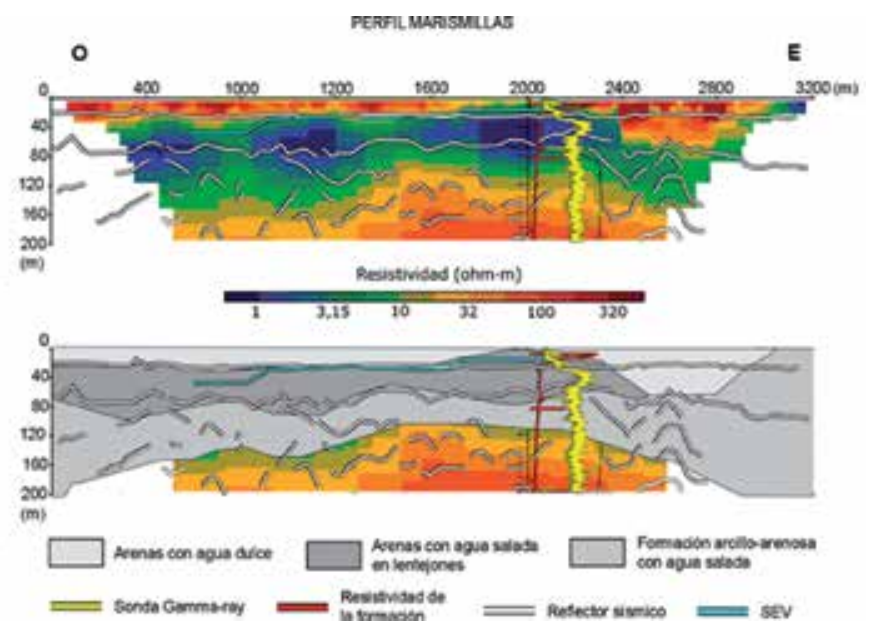

Figura 14. Superior: Superposición de la interpretación de un SEV próximo, testificación del sondeo mecánico y de los reflectores sísmicos sobre la sección geoeléctrica. Inferior: esquema de Interpretación geológica del perfil de Palacio de las Marismillas.

Figure 14. Upper: Composition over the geoelectric section of the interpretation of a nearest VES, a log from a well and seismic reflectors. Bottom: Scheme of the Geological interpretation of Palace of Marismillas electrical profile.

sobre todo las superficiales, que presentan las secciones geoeléctricas (Figura 13). No obstante, aunque el ruido puede afectar de forma local a la interpretación, no produce grandes distorsiones en las tendencias generales, por lo que se estima satisfactoria la interpretación general expuesta.

En la zona del Palacio de Marismillas se trató de estudiar el contacto entre las aguas salobres y el agua dulce en las dunas de arena situadas al suroeste. En la testificación de resistividad de un sondeo próximo (S-59) se observa que existe un aumento de salinidad con la profundidad. Esto justifica los bajos valores de resistividad registrados con la sonda de inducción, en constante disminución con la profundidad. Sin embargo, el hecho de que un pequeño nivel de margas a los $78 \mathrm{~m}$ quede reflejado en el registro de resistividad de la formación, induce a pensar que el agua salada que existe dentro de la columna del sondeo no está presente en toda la formación litológica, ya que entonces hubiera imposibilitado detectar este contraste.

Se efectuaron Sondeos Electromagnéticos en el Dominio del Tiempo (SEDT) y Sondeos Eléctricos Verticales (SEV) que indican una continuidad de las unidades geoeléctricas, deduciéndose que toda la zona contiene agua salinizada; sin embargo la inversión de los datos medidos presenta importantes ambigüedades, por lo que los resultados no son del todo coherentes. En el perfil continuo de resistividad
(Figura 14) se detecta una cobertera resistiva, que coincide con la medida por los SEV, por los SEDT y por la testificación. Por debajo se sitúa una capa muy conductora, también registrada en los SEDT y SEV. Finalmente, por debajo de los $100 \mathrm{~m}$ de profundidad se detecta una capa más resistiva, no vista en los SEV, ni en los SEDT, ni en el registro de resistividad, y que puede considerarse como un falso resultado de la inversión, no siendo la sección válida por debajo de unos $120 \mathrm{~m}$.

Por otra parte, es posible apreciar una correspondencia entre facies sísmicas y eléctricas (Figura 14). EI primer reflector marca la base del tramo inicial resistivo (>20 ohm.m), que según la columna del sondeo se trata de arenas. El segundo reflector marca la base de un primer tramo arcilloso, de menos de 2 ohm.m, reconocido por la testificación gamma natural.

En el extremo oriental, este reflector marca el paso del resistivo de más de 200 ohm.m a un tramo más conductor, en total coincidencia con la sección geoeléctrica. El segundo tramo de reflectores se sitúa en la zona de terrenos con mayor resistividad, que según la testificación tienen menor contenido en arcillas. En la Figura 14 se representa un esquema geológico-conceptual de este perfil, que incluye el aludido tramo resistivo de existencia poco probable al no presentar correlación con el resto de técnicas aplicadas. Este esquema constituye la conclusión obtenida a partir de tomografía eléctrica para el objetivo planteado en esta zona sobre la superficie del contacto de las aguas salobres y las dunas de arena, donde el agua es dulce (procedente de la recarga de lluvia).

Se puede concluir que las arcillas de la zona del Palacio de Marismillas están fuertemente salinizadas, encontrándose a menor profundidad en la zona del sondeo mecánico. Este modelo está respaldado por las interpretaciones de los SEV, SEDT, el perfil eléctrico continuo y la sección sísmica. A nivel metodológico se concluye que los perfiles eléctricos son una buena opción para abordar esta clase de problemáticas, resultando la técnica geofísica más satisfactoria de entre las ensayadas.

La tomografía eléctrica, por tanto, ha resultado ser un método eficaz para determinar contactos litológicos en profundidad entre unidades de diferente resistividad incluso bajo el nivel freático. Sin embargo no ha resultado tan efectivo cuando existen heterogeneidades laterales. Asimismo ofrece resultados satisfactorios para caracterizar la geometría del contacto entre aguas con fuertes contrastes de salinidad, si bien no es posible distinguir entre distintas litologías debido a que el agua salada enmascara los contrastes de resistividad litológicos si éstos no son muy 
pronunciados. Del mismo modo, allí donde existen fuertes contrastes de resistividad debido a las distintas litologías, resulta dificultoso distinguirlos entre masas de agua de distinta salinidad.

\section{Caso 3. La laguna de Fuente de Piedra (Málaga)}

Otro de los espacios singulares estudiados ha sido la laguna de Fuente de Piedra al noroeste de la provincia de Málaga (Figura 1). Es un reservorio de agua salada que se encuentra en el interior de una cuenca endorreica ubicada entre las cuencas hidrográficas de los ríos Guadalquivir (al Norte) y Guadalhorce (al Sur). El interés medioambiental de la laguna es notorio, ya que contiene comunidades de plantas halófilas y es lugar de paso de aves migratorias. Está calificada como Reserva Natural, y está incluida en la lista de humedales españoles incorporados al Convenio de Ramsar. Esta singularidad ha motivado el desarrollo de diversos estudios dirigidos a caracterizar su funcionamiento hidrogeológico, desde la década de los 80 (ITGE 1983, 1984 y 1998).

La zona se caracteriza por la presencia de materiales triásicos arcillosos, yesíferos y salinos, que sufren una intensa karstificación y dan lugar a procesos de colapso y hundimiento. Estratigráficamente por encima de estos materiales se encuentra una serie miocena de calcarenitas, arenas y margas con espesores de hasta cien metros. Estos materiales aparecen distribuidos de forma irregular en amplias zonas, que incluyen acuíferos y lagunas, con diverso grado de salinidad, originados por la disolución de los materiales yesíferos y salinos del Keuper. La laguna de Fuente de Piedra y su cuenca constituyen uno de los mayores sistemas de salmueras de este complejo. Debido al carácter endorreico de la cuenca es posible encontrar agua dulce en las formaciones de calizas junto a salmueras de alta concentración, superior en zonas profundas a los $330 \mathrm{~g} / \mathrm{l}$, y zonas salobres de distinto grado de salinidad (Heredia et al., 2007, 2009).

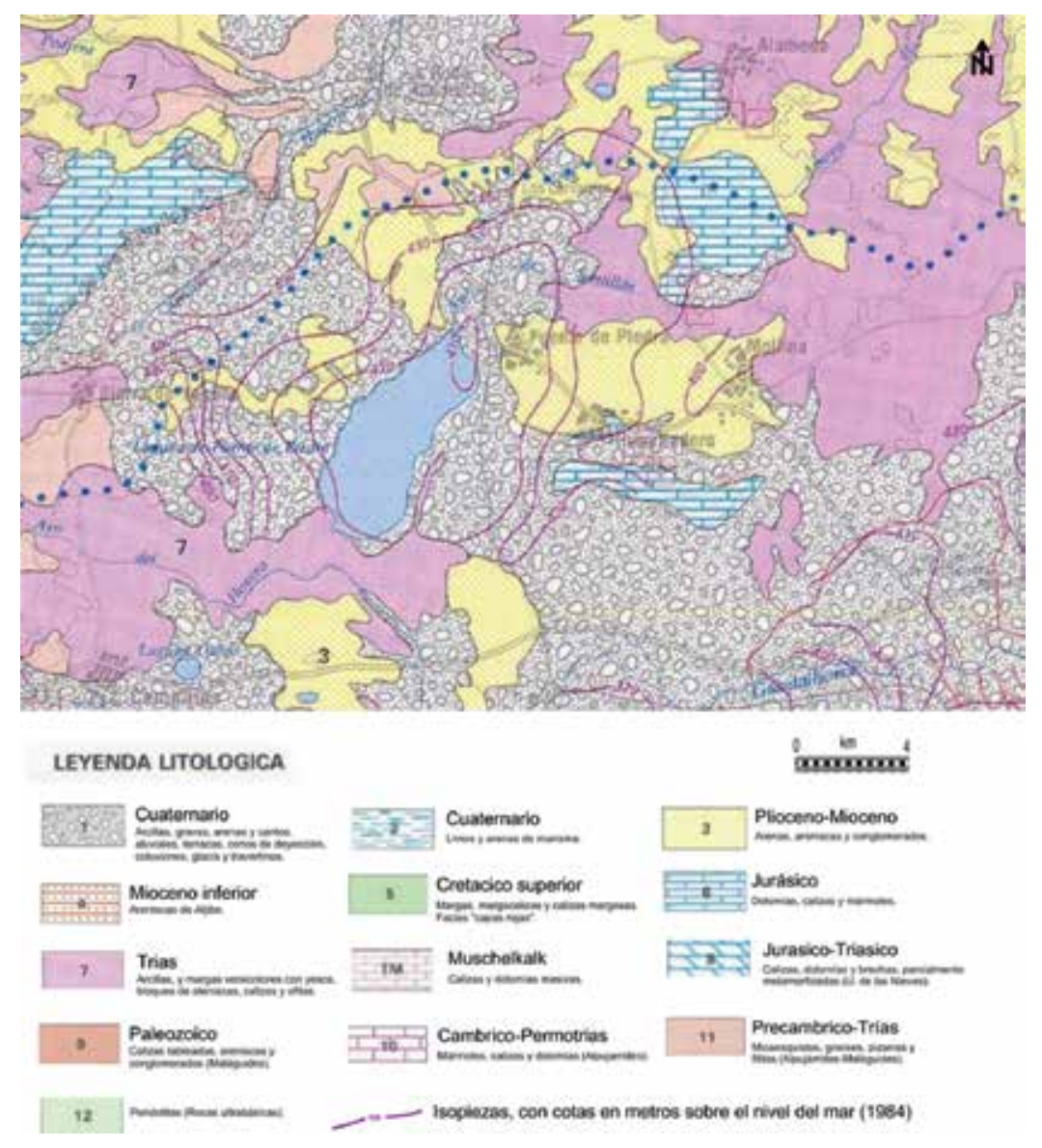

Figura 15. Recorte del mapa Hidrogeológico a escala 1:200000 $\mathrm{n}^{\circ} 82$ Morón de la Frontera en la zona de la laguna de Fuente de Piedra. Figure 15. Detail of the 1: 200000 Hydrogeological map $n^{\circ} 82$ Morón de la Frontera including the lagoon of Fuente de Piedra. 
El humedal está emplazado en una depresión en superficie asociada a uno de estos procesos de karstificación producido en profundidad, que da lugar a la acumulación endorreica del agua de la laguna. Los procesos de disolución de yesos y sales, unidos a la evaporación de la laguna, han dado lugar al desarrollo de varias salmueras. Esta configuración geológica ha dado lugar a tres tipos de acuífero: el inferior, de tipo kárstico, formado por sales y yesos; el intermedio, de tipo intergranular, emplazado en las calcarenitas miocenas; y el superior, también de tipo intergranular, localizado en los sedimentos cuaternarios (Figura 15). Estos acuíferos presentan un diferente comportamiento hidrogeológico, pero aparentemente están interconectados (Heredia et al., 2009).

Entre los años 2005 y 2006 el IGME llevó a cabo un estudio, en el que la geofísica demostró ser de gran utilidad al proporcionar datos sobre la ubicación de las salmueras y su alcance, extensión, e incluso concentración de sales, lo que resultó fundamental para el desarrollo de un modelo hidrogeológico que contribuyera a la mejora de la gestión hídrica de la cuenca (Heredia et al., 2009).

En el año 2005 se realizó una campaña de tomografía eléctrica, diseñada a modo de ensayo de viabilidad del método para la detección de salmueras (Ruiz et al., 2006; Heredia et al., 2009). La testificación geológica y geofísica de varios pozos de control sirvió para correlacionar las variaciones de resistividad de los perfiles con la presencia de salmueras, si bien no fue posible emplear estas variaciones de resistividad

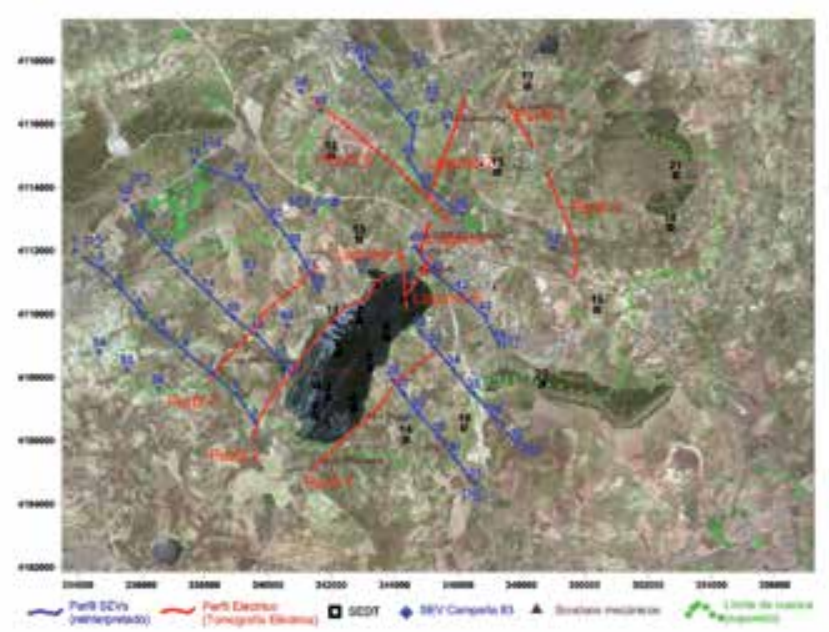

Figura 16. Situación sobre foto de satélite de los trabajos geofísicos realizados la zona de la Laguna de Fuente de Piedra (Cartografía y PNOA del Instituto Geográfico Nacional, Andalucía).

Figure 16. Position of the surface geophysical works carried out in the lagoon of Fuente de Piedra over a satellite photo (Cartography and PNOA from Instituto Geográfico Nacional, Andalucía). para diferenciar las distintas unidades geológicas. Se midieron 4 perfiles en el borde norte e interior de la laguna y en la zona norte de la cuenca (denominados Laguna en la Figura 16). En su realización se utilizaron diversos espaciados interelectródicos (5 y $15 \mathrm{~m})$ con el objetivo de investigar a diferentes profundidades. Su ubicación se decidió en función de los pozos de investigación disponibles de modo que fuera posible establecer un criterio de correlación entre la testificación geológica, testificación geofísica y el perfil eléctrico.

Los resultados de este estudio motivaron una nueva campaña de investigación en 2006, en la que se midieron 6 perfiles distribuidos por toda la cuenca, que cubrieron $25 \mathrm{~km}$ más respecto a la campaña anterior (Ruiz et al., 2006; lbarra et al., 2008; Heredia et al., 2009). Complementariamente, entre 2005 y 2006 se han realizado un total de 11 SEDT en el interior del vaso de la laguna y zonas marginales de ésta, y 10 más en puntos dispersos de la cuenca endorrei$\mathrm{ca}$, realizados en zonas no cubiertas previamente por otros métodos (Ibarra et al., 2006; Heredia et al., 2009), lo que permitió alcanzar una mayor profundidad de investigación que la obtenida con los perfiles eléctricos así como cubrir zonas de difícil acceso en las que no se habían realizado mediciones mediante tomografía eléctrica. Por otra parte se reinterpretaron los Sondeos Eléctricos Verticales (SEV) procedentes de una campaña previa del año 1983 (ITGE, 1983), generando nuevos perfiles, lo que permitió densificar y complementar la información geofísica de la cuenca.

En la Figura 17 se muestra el resultado de la inversión de algunos de los perfiles eléctricos realizados. El perfil Laguna 3, de $1200 \mathrm{~m}$ de longitud medido parcialmente en el interior del vaso de la laguna, con dirección NE-SO, y con espaciado interelectródico de $15 \mathrm{~m}$. Este perfil presenta un bloque muy conductor en niveles superficiales, claramente atribuible al acuífero salino que se ubica bajo la laguna así como en los terrenos inundables que se sitúan en el norte de ésta (entendiendo por inundables las zonas bajas que antaño se anegaban con cierta frecuencia fuera del vaso actual). Se aprecia también, la existencia de un nivel relativamente resistivo con valores de hasta $10 \mathrm{ohm} . \mathrm{m}$ que profundiza notablemente a la altura del límite de la laguna y dentro de ésta. Es decir, bajo la laguna el cuerpo resistivo tiene mayor profundidad, si bien el cambio de resistividad es muy tenue y resulta complicado afirmar su continuidad (línea discontinua en la Figura 16). Lo más notable por tanto, es la existencia de un nivel resistivo, análogamente a lo encontrado en otros perfiles medidos en el vaso de la laguna, que podría representar algún tipo de control litológico. También es remarcable el salto en profundidad de este cuerpo resistivo, brusco y vertical, 


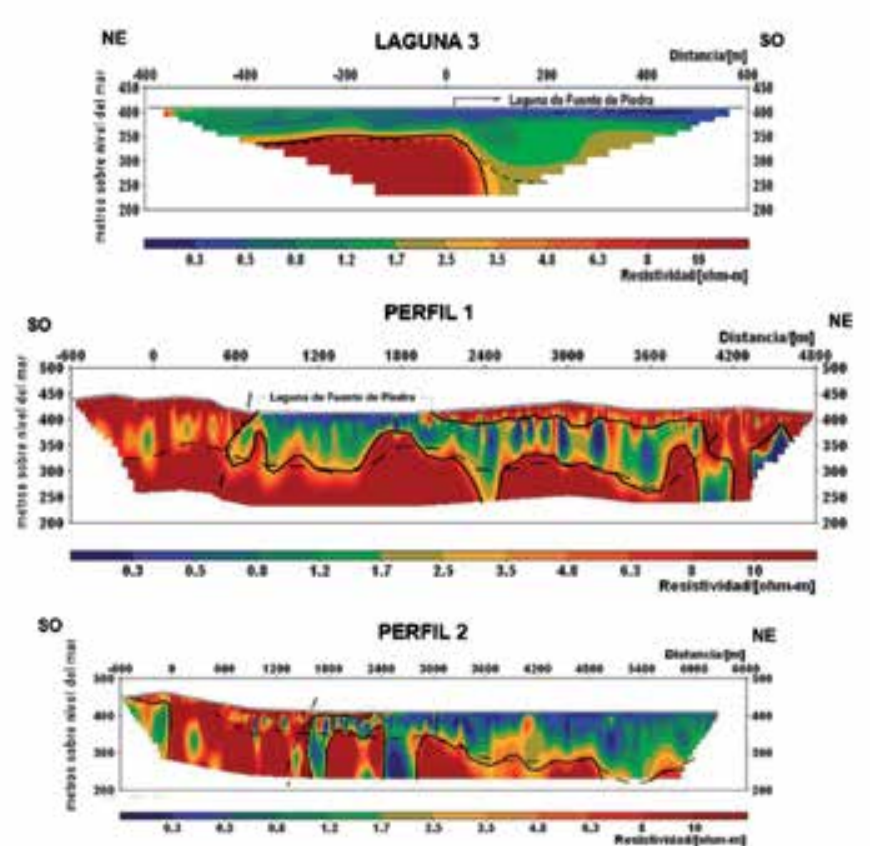

Figura 17. Superior: Perfil Laguna 3. Centro: Perfil 1 largo. Inferior: Perfil 2 largo. Límite de la salmuera en trazo continuo. Límite de la unidad geológica en trazo discontinuo.

Figure 17. Top: Laguna 3 electrical profile. Center: Perfil 1 long electrical profile. Bottom: Perfil 2 long electrical profile. Continuous line indicates the limit of the brine. Dashed line indicates the limit of the geological unit.

que parece estar relacionado con el límite de la propia laguna.

En el Perfil 1 (Figura 17 centro) se puede observar bajo la laguna de Fuente de Piedra un cuerpo de muy baja resistividad (entre 0 y 2 ohm.m) que se interpreta como la masa de salmueras. Esta masa se extiende en mayor medida al NE que al SO, y presenta embolsamientos inconexos de muy baja resistividad. En este perfil hacia el NE se observa una prolongación en profundidad del cuerpo de salmueras, lo que sugiere la presencia de salmueras más profundas o que su génesis se produce a partir de niveles más profundos, pudiendo existir un flujo vertical a través de estos niveles.

El Perfil 2, medido en el lado Oeste de la laguna de Fuente de Piedra, es un ejemplo de sección realizada fuera de los límites de la cuenca endorreica hasta alcanzar tangencialmente el borde oeste de la laguna (Figura 17 inferior). La longitud del perfil es de $6900 \mathrm{~m}$, con espaciado entre electrodos de $15 \mathrm{~m}$. EI perfil presenta un tramo inicial en el que se observan materiales conductores aislados, atribuibles a cuerpos individuales de salmueras, y un segundo tramo en el que predominan los materiales menos resistivos, atribuibles a las mayores masas de salmueras conectados con la laguna. El cuerpo de salmueras del segundo tramo de perfil presenta una estructura irregular hacia su base, con entrantes en profundidad localizados en las posiciones 1750 y $2500 \mathrm{~m}$ del perfil

De la interpretación de los datos geofísicos, especialmente los perfiles eléctricos, se ha extraído que el cuerpo de salmuera asociado a la laguna tiene una extensión mayor que el tamaño actual del vaso de la misma, especialmente al norte, oeste y sureste de la laguna. En general, el conductor se va adelgazando y profundizando conforme aumenta la distancia a la laguna a modo de cuña. Bajo el vaso de la laguna se observa un gran nivel conductor, que es asimilable a un nivel saturado de salmueras, limitado por debajo, por un nivel de mayor resistividad relativa que parece actuar como un control litológico y que se presenta a profundidad variable, en el entorno de los 60-100 m por debajo de la laguna, aunque en algunos casos es superior. Este nivel presenta un resalte de resistividad coincidente con el límite del vaso de la laguna actual, lo que podría responder a algún tipo de colapso de los materiales karstificados sobre los que se asienta la laguna, efecto que se aprecia sobre todo en su límite Norte. El lado sur de la laguna, parece presentar un claro control geológico que limita la extensión de las salmueras en esa dirección.

De la interpretación conjunta de las diferentes campañas geofísicas efectuadas, se han extraído las siguientes conclusiones (Heredia et al., 2009): en el conjunto de la cuenca se identifican numerosos cuerpos conductores, con diversa extensión, atribuibles a la presencia de salmueras, de mayor o menor concentración. Estos cuerpos están repartidos de forma irregular en toda la cuenca e incluso fuera de ella y son de continuidad y distribución inhomogéneas. Estos cuerpos son de morfología más o menos lenticular, y suelen estar ubicados a profundidades medias (de 50 a $100 \mathrm{~m}$ ), a modo de un confinamiento finito que parece implicar un escaso movimiento de las salmueras. Suelen tener una base a modo de control geológico, con profundidades variables, Figura 17 perfil 1.

Lejos de la laguna, los horizontes son en general de carácter más resistivo. Sin embargo, aparecen igualmente de forma irregular o diseminada algunos niveles conductores que estarían ocupados por salmueras con diferentes grados de concentración.

Algunos cuerpos conductores tienen extensiones mayores en profundidad con respecto al sugerido nivel de control. Se trata de cuerpos con morfologías más verticales, con morfologías globulares de salmueras de gran continuidad en profundidad cuyo límite inferior es desconocido; esto sugiere la existencia de salmueras ubicadas a mayor profundidad, cuya base no ha sido posible identificar con la 
metodología empleada (debido a las limitaciones de profundidad de investigación y al carácter individualizado de estos conductos, que no presentan una extensión suficiente para ser detectados con claridad); ejemplos de los mismos pueden observarse en la Figura 17 en el perfil 1 y perfil 2. Esto sugiere la existencia de algún tipo de fenómeno convectivo en los niveles de salmueras, creando ciertas zonas de circulaciones profundas preferentes a modo de celdas.

Los patrones identificados en la distribución de salmueras parecen responder a dos modelos diferentes. Un primer modelo en el que existe un gran acuífero asociado a la laguna, cuyos límites trascienden a los de ésta hasta acuñarse a cierta distancia sujeta a controles geológicos. El segundo modelo responde a una serie de acumulaciones de salmueras por toda la cuenca con diverso grado de aislamiento, continuidad y conectividad entre sí, lo que denota un aparente escaso flujo entre ellas. En ambos casos, parecen existir eventuales conexiones de salmueras con niveles más profundos, que pudieran implicar flujos verticales entre unos y otros, o un origen más profundo de las salmueras.

El método de tomografía eléctrica ha permitido definir la disposición de los niveles de salmueras, lateralmente y en profundidad. Esto resultó de gran interés para mejorar el conocimiento del funcionamiento hidrogeológico de la laguna de Fuente de Piedra, y así contribuir a la preservación de ésta y de los ecosistemas asociados. La tomografía eléctrica se complementó satisfactoriamente con los datos procedentes de SEV y SEDT, ofreciendo todos ellos resultados coherentes y compatibles entre sí, lo que refuerza la fiabilidad de las técnicas aplicadas (Heredia et al., 2009).

\section{Caso 4. El Paleolago de La Larri}

El último caso presentado corresponde al de Paleolago de La Larri (Figuras 1 y 18). El valle de La Larri se aloja lateralmente sobre la cabecera del valle de Pineta (alto río Cinca, en el límite nororiental del Parque Nacional de Ordesa y Monte Perdido), en el contexto de una ventana tectónica abierta por la actividad erosiva de un glaciar lateral al principal de Pineta (Pérez-Bielsa, 2013). La actividad glaciar ha afectado a la geomorfología de toda la región, y en particular al valle de La Larri, cuyo río presenta un trazado que es combinación de las acciones erosivas fluviales y glaciares. Este emplazamiento resulta de singular importancia para el estudio de la cronología de la máxima extensión de los hielos durante el último ciclo glaciar y las posteriores fases de deshielo

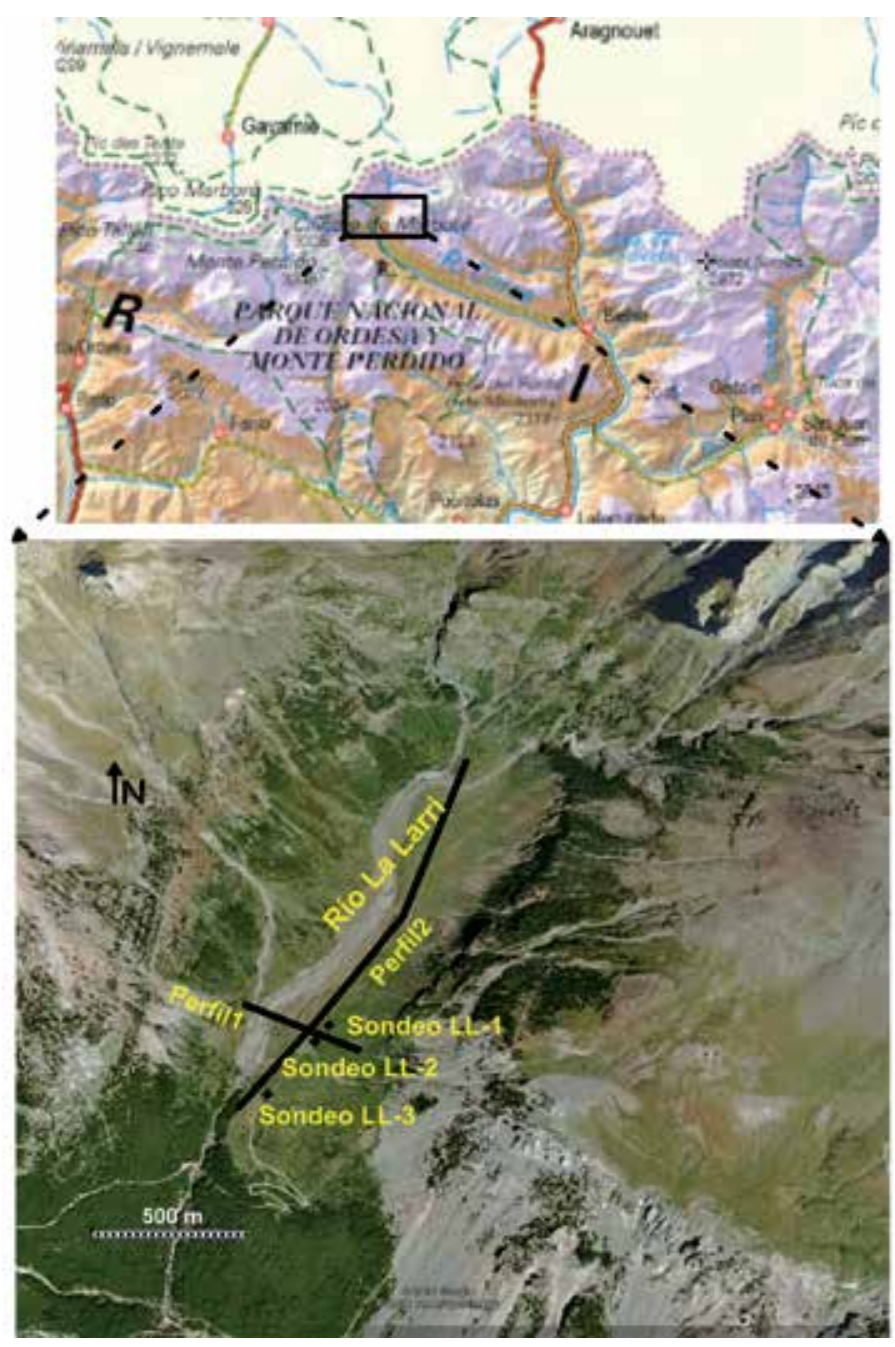

Figura 18. Situación del valle de La Larri, y posición de los perfiles y sondeos mecánicos sobre imagen de satélite. (Cartografía y PNOA del Instituto Geográfico Nacional, Aragón).

Figure 18. Situation of the La Larri valley and position of the ERT profiles and wells carried out in the valley over satellite image. (Cartography and PNOA from Instituto Geográfico Nacional, Aragón).

(Martí-Bono and García-Ruiz, 1994; Calvet, 2004; González-Sampériz et al., 2006), particularmente debido a la diacronía existente entre los últimos máximos glaciares, local y global, en las áreas de montaña en el entorno del mediterráneo (Hughes and Woodward, 2008).

En el valle de La Larri existen depósitos sedimentarios glacio-lacustres que estarían relacionados con las fases glaciares posteriores al último máximo glaciar local (Salazar-Rincón et al., 2013), por lo que resultan de gran interés para el estudio de los ciclos glaciares en la zona. Dos aspectos fundamentales que es necesario conocer son el espesor y la 
extensión de estos rellenos. En primer lugar, de ello dependerá la resolución y el lapso temporal abarcado por el registro sedimentario, y por lo tanto el alcance de la información disponible y de las interpretaciones que de ella se deriven. Por otra parte, el espesor de sedimentos está relacionado con la duración de la actividad del medio sedimentario en el que fueron depositados, lo que resulta de gran interés para conocer la cronología de la actividad glaciar.

Los objetivos del estudio geofísico fueron definir el tipo y espesor del relleno sedimentario del Paleolago de La Larri, para evaluar la duración del registro sedimentario y la distribución de las distintas facies sedimentarias. Los testigos obtenidos en sondeo ofrecían información precisa acerca de la litología y espesor de las unidades en la ubicación de la perforación, sin embargo era también necesario conocer la geometría lateral de los cuerpos sedimentarios para construir el modelo geológico. Además, una de las principales limitaciones de la información proporcionada por los sondeos es la escasa profundidad que éstos alcanzaron, por lo que no se conoce con exactitud las litologías subyacentes a las últimas representadas en los testigos.

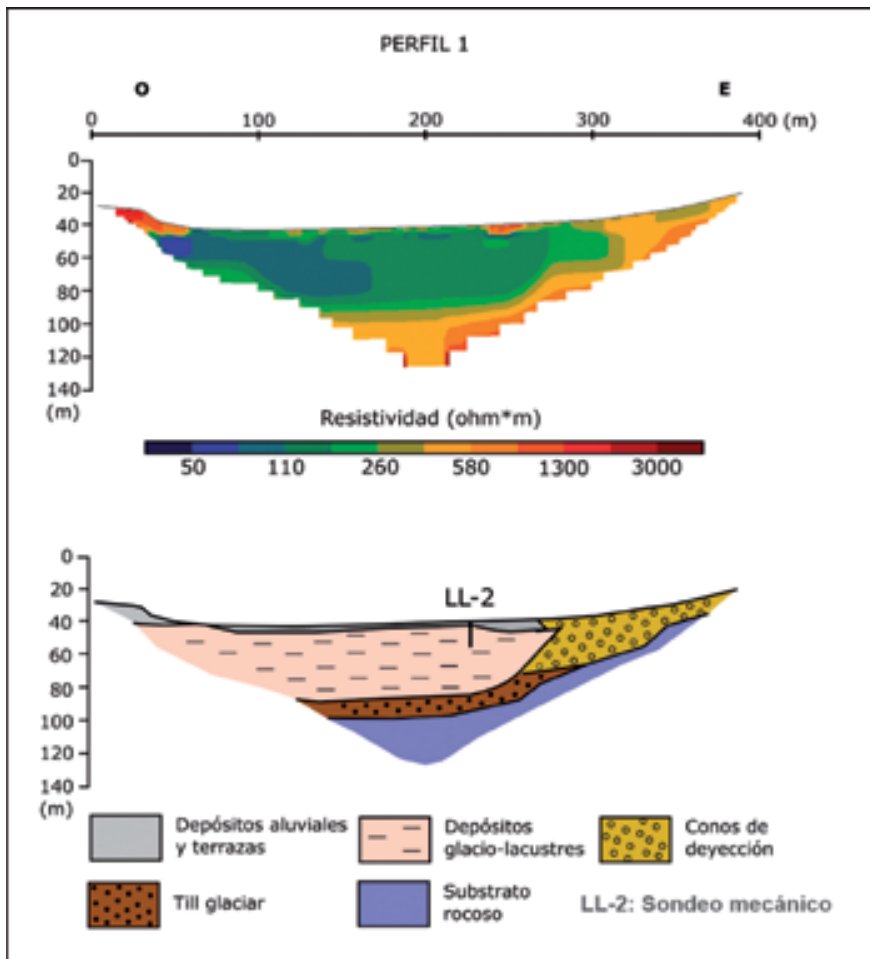

Figura 19. Arriba: perfil de tomografía eléctrica transversal al valle de La Larri. Abajo: interpretación geológica. Adaptado de SalazarRincón (2013).

Figure 19. Up: ERT profile across the lower section of valley of $\mathrm{La}$ Larri. Down: geological interpretation, from Salazar-Rincón (2013).
Se realizaron dos perfiles de tomografía eléctrica (Figura 18) que se integraron con la información recogida en sondeos mecánicos. El perfil 1 (Figura 19), fue medido transversal al paleovalle, con una distancia interlectródica de $5 \mathrm{~m}$ y empleando un dispositivo Wenner; el perfil 2 (Figura 20) fue medido a lo largo del paleovalle, con un espaciado intereléctródico de $15 \mathrm{~m}$ y también con dispositivo Wenner, de modo que la profundidad de penetración fuera del orden de 100 $\mathrm{m}$, mayor que la alcanzada por los sondeos, y alcanzase el substrato rocoso resistivo bajo los sedimentos glaciares.

La interpretación del perfil 1 (Figura 19) presenta un relleno conductor, asociado a lutitas glacio-lacustres, que supera los $60 \mathrm{~m}$ de potencia y que son en los que penetra el sondeo LL-2. En la parte oriental se observa el paso lateral hacia materiales más resistivos asociados a conos de deyección. En la parte occidental el material resistivo final corresponde a la terraza aluvial.

Los resultados obtenidos en el perfil 2 (Figura 20) muestran la presencia de un cuerpo conductor asociado a los materiales de relleno del valle (depósitos glacio-lacustres); hacia la base de estos materiales,

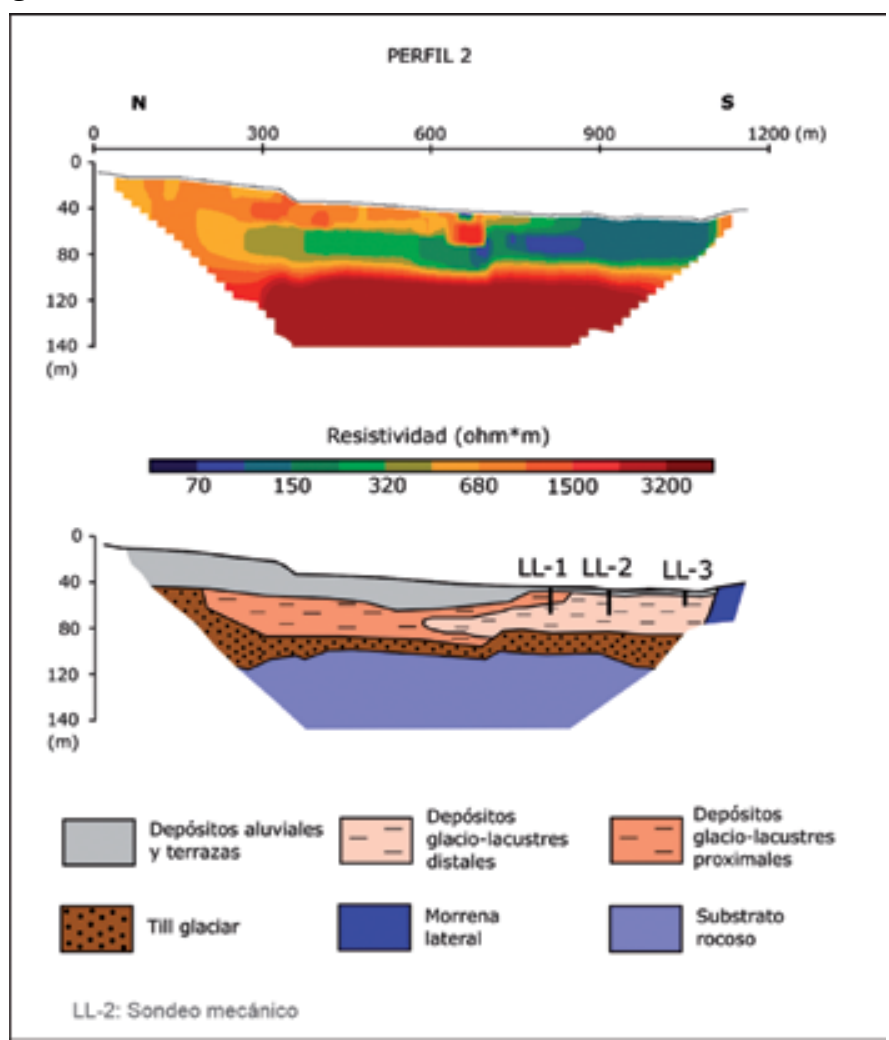

Figura 20. Arriba: perfil de tomografía eléctrica longitudinal al valle de La Larri. Abajo: interpretación geológica. Adaptado de SalazarRincón (2013).

Figure 20. Up: ERT profile along the Valley of La Larri. Down: geological interpretation from Salazar-Rincón (2013). 
Rubio Sánchez-Aguililla, F.M., et al., 2017. Métodos geofísicos en entornos naturales... Boletín Geológico y Minero, 128 (1): 171-192

existe una zona de transición que se ha asociado a un till glaciar, el cual descansaría sobre el sustrato rocoso inalterado asociado al cuerpo fuertemente resistivo más profundo. En el extremo este del perfil, existe un cuerpo de carácter resistivo que se ha interpretado como un cono de deyección y en el extremo oeste se muestra un cuerpo resistivo que corresponde a la morrena lateral del valle de Pineta que sirvió como represa del paleolago de La Larri.

En este caso de aplicación, el método de tomografía eléctrica ha resultado útil para identificar las distintas litologías que componen los sedimentos del fondo del valle de La Larri, debido a que presentaban contrastes de resistividad suficientes. La integración de los datos de los perfiles eléctricos con la testificación litológica de los sondeos existentes ha permitido afianzar las interpretaciones de los perfiles, apoyando la correlación entre las distintas unidades litológicas y geoeléctricas. El estudio permitió conocer la geología del subsuelo ofreciendo información continua tanto en profundidad como lateralmente, lo que contribuyó a mejorar el conocimiento de los ciclos glaciares locales en la zona. Ampliar el espaciado interelectródico y, por lo tanto, la longitud de la implantación permitió alcanzar mayores profundidades de investigación, y la pérdida de resolución asociada no impidió interpretar la distribución de los depósitos sedimentarios, separando las fases de sedimentación lacustre y de sedimentación glaciar. La tomografía eléctrica permitió investigar a una mayor profundidad sin realizar nuevos sondeos.

\section{Conclusiones}

La tomografía eléctrica se presenta como una herramienta de estudio eficaz para resolver cuestiones de carácter geológico e hidrogeológico, tanto como único método de investigación o combinado con otros métodos. El conocimiento de la distribución de resistividad en el subsuelo permite identificar la posición y la morfología de distintas unidades geológicas en función de su litología e hidrogeológicas en función de la salinidad del fluido.

En combinación con los sondeos de resonancia magnética y la tomografía eléctrica permitió mejorar el conocimiento del acuífero de las lagunas de Estaña (Huesca), aportando información sobre su extensión lateral y en profundidad, así como diferenciar las distintas unidades hidrogeológicas que lo componen. Todo ello supuso una mejora del conocimiento sobre su funcionamiento hidrogeológico, contribuyendo a delimitar los factores clave que controlan el sistema y, por lo tanto, a su conservación.
En el acuífero Almonte-Marismas (Parque Nacional de Doñana) se pudo definir la geometría del contacto entre las dunas y la marisma a partir de los perfiles eléctricos, pudiendo distinguir sub-unidades de distinta resistividad tanto en las dunas como en la marisma. Se observó una buena correspondencia entre los perfiles eléctricos y los perfiles sísmicos. EI estudio aportó nueva información sobre el funcionamiento hidrogeológico del sistema, lo que permitirá optimizar el uso de los recursos hídricos en la zona protegiendo los ecosistemas asociados a él.

Asimismo, ha sido posible determinar la extensión y espesor de los cuerpos de salmueras asociados a la laguna de Fuente de Piedra (Málaga), que presentan una morfología de bolsadas interconectadas. Los perfiles obtenidos sugieren un origen profundo de las salmueras y la posible existencia de flujos verticales. Estas investigaciones permitieron profundizar más en el conocimiento de este sistema singular de gran importancia ecológica.

Por último en el valle de La Larri, los perfiles eléctricos permitieron identificar las distintas unidades sedimentarias y estimar el espesor de depósitos glacio-lacustres, ampliando la información proporcionada por los sondeos preexistentes en la zona. Esto contribuyó a mejorar el conocimiento de los ciclos glaciares en la zona sin necesidad de realizar nuevos sondeos, alcanzando mayores profundidades de investigación y proporcionando información continua lateralmente.

Con ello queda patente la capacidad de la tomografía eléctrica para proporcionar resultados satisfactorios en entornos en los que son esperables grandes contrastes de resistividad en el subsuelo, permitiendo identificar las unidades geológicas o hidrogeológicas clave. La tomografía eléctrica es un método de bajo coste, rápida aplicación y reducido impacto en el terreno, lo que reduce la necesidad de realizar sondeos para abordar el estudio. Esto permite investigar el subsuelo en áreas de elevada importancia ambiental y/o patrimonial, ofreciendo nuevas posibilidades para mejorar el conocimiento de estas áreas singulares y así contribuir a su conservación y explotación sostenible en el tiempo.

\section{Referencias}

Abem. 2010. Terrameter SAS 4000/ SAS 1000 manual instruction, 23/6/2015, http://www.abem.se/support/ downloads/user-manuals/manual_terrameter.pdf

Ball, L.B., Ge, S., Caine, J.S., Revil, A. y Jardani, A. 2010. Constraining fault-zone hydrogeology through integrated hydrological and geolelectrical analysis. Hydrogeology Journal, 18 (5), 1057-1067. 
Calvet, M. 2004. The quaternary glaciation of the Pyrenees. In J. Ehlers, P.L. and Gibbard (eds). Quaternary Glaciations - Extent and Chronology. Part I: Europe. Elsevier, Amsterdam, 119-128

Chambers, J.E., Kuras, O., Meldrum, P.I., Ogilvy, R.D. y Hollands J. 2006. Electrical resistivity tomography applied to geologic, hydrogeologic, and engineering investigations at a former waste-disposal site. Geophysics, 71 (6), 231-239

CHE. 2002. Estudio de recursos hídricos subterráneos de los acuíferos de la margen izquierda de la cuenca del Ebro. Zona oriental. Litera Alta (09.304). Informe inédito. Confederación Hidrográfica del Ebro, Zaragoza.

Edwards, L.S. 1977. A Modified Pseudosection for Resistivity and Induced Polarization. Geophysics. 42, 1020-1036.

Galazoulas, C. E., Mertzanides, C.Y., Petalas, C.P. y Kargiotis, E. K. 2015. Large scale electrical resistivity tomography survey correlated to hydrogeological data for mapping groundwater salinization: a case study from a multilayered coastal aquifer in Rhodope, Northeastern Greece. Environmental processes, 2 (1), 19-35.

Geotomo, 2015. Manual del software res2dinv 23/6/2015 http://www.geotomosoft.com/r2dimanu.zip

Griffiths, D.H. y Barker, R.D. 1993. Two-Dimensional Resistivity Imaging and Modeling in areas of Complex Geology. Journal of Applied Geophysics, 29, 211-226.

González-Sampériz, P., Valero-Garcés, B.L., Moreno, A., Jalut, G., García-Ruiz, J.M., Martí-Bono, C., DelgadoHuertas, A., Navas, A., Otto, T. y Dedoubat, J.J. 2006. Climate variability in the Spanish Pyrenees during the last $30,000 \mathrm{tr}$ revealed by $\mathrm{El}$ Portalet sequence. Quaternary Research. 66, 38-52

Heredia, J., Ruiz, J.M., García de Domingo, A., Rubio, F.M. y Ibarra, P. 2007 Empleo de técnicas geofísicas en la caracterización de la cuenca endorreica de Fuente de Piedra, Málaga (España). Sosa, D., and Ainchil, J. (ed.), Taller de Geofísica aplicada a la hidrogeología, $\checkmark$ Congreso Argentino de Hidrogeología. III Seminario Hispano- Latinoamericano de Hidrología Subterránea. Paraná (Argentina). 95-104

Heredia, J., García de Domingo, A., Ruiz, J.M., Ibarra, P. y Rubio F.M. 2009. Caracterización hidrogeológica y modelización numérica de un sistema de flujo con densidad variable: sistema hidrogeológico de la laguna de Fuente de Piedra (Málaga). Informe inédito. Centro de Documentación del IGME, Madrid. N F.D. 63833, 169 págs + anexos

Hughes, P.D. y Woodward, J.C. 2008. Timing of glaciation in the Mediterranean mountains during the last cold stage. Journal of Quaternary Science, 23 (6-7), 575-588.

Ibarra, P., Fernández, I., Verges, Y. y Mora-Pérez, E.E. 2006. Estudio mediante Sondeos Electromagnéticos en el Dominio de Tiempos con fines hidrogeológicos en la Laguna de Fuente de Piedra (Malaga). Informe inédito. Centro de Documentación del IGME, Madrid. N F.D. 63358, 33 págs. + anexos

Ibarra, P., Rubio, F.M., Heredia, J., Ruiz, J.M., y G. de Domingo, A. 2008. Geofísica combinada para modelización hidrogeológica en Fuente de Piedra. Resúmenes $6^{a}$ Asamblea Hispano-Portuguesa de Geodesia y Geofísica. Tomar (Portugal), 295-296.

IGME, 1994. Hoja de Fonz (288). Mapa Geológico de España (E. 1:50000), 89 págs.

IGME, 1995. Hoja de Benabarre (289). Mapa Geológico de España (E. 1:50000), 191 págs.

IGME, 2007. Hoja de Os de Balaguer (327). Mapa Geológico de España (E. 1:50000), 113 págs.

ITGE. 1983. Estudio geoeléctrico en Fuente de Piedra. Malaga. Informe inédito. Centro de Documentación del IGME, Madrid. N F.D. 40231, 127 págs.

ITGE. 1984. Estudio Hidrogeológico para conservación de la naturaleza en cuenca del sur de España y Canarias: Laguna de Fuente de Piedra. Informe inédito. Centro de Documentación del IGME, Madrid. N F.D. 31135, 346 págs.

ITGE. 1998. Hidrogeología de la reserva natural de la Laguna de Fuente de Piedra (Malaga). Informe inédito. Centro de Documentación del IGME, Madrid. $N^{\circ}$ F.D. 31206

Lago, M. y Pocovi, A. 1982. Nota preliminar sobre la presencia de estructuras fluidales en las ofitas del área de Estopiñán (provincia de Huesca), Acta Geológica Hispánica, 17, 4, 227-233

Loke, M.H. 1999. Electrical imaging surveys for environmental and engineering studies. A practical guide to 2-D and 3-D surveys. 23/7/2015, http://www.geomatrix. co.uk/tools/application-notes/Lokenote.pdf

Martínez-Pagán, P., Gómez-Ortiz, D., Martín-Crespo, T., Manteca, J.I. y M. 2013. The electrical resistivity tomography method in the detection of shallow mining cavities. A case study on the Victoria Cave, Cartagena (SE Spain). Engineering Geology, 156, 1-10.

Martinez-Moreno, F.J., Pedrera, A., Ruano, P., GalindoZaldivar, J., Martos-Rosillo, S., Gonzalez-Castillo, L., Sánchez-Ubeda, J.P. y Marín-Lechado, C. 2013. Combined microgravity, electrical resistivity tomography and induced polarization to detect deeply buried caves: Algaidilla cave (Southern Spain). Engineering Geology, 162, 67-78.

Martí-Bono, C. y García-Ruiz, J.M. (eds). 1994. El Glaciarismo Surpirenaico: nuevas aportaciones. Geoforma Ediciones, Logroño, 142 pp

Meads, L.N., Bentley, L.R. y Mendoza, C.A. 2003. Application of electrical resitivity imaging to the development of a geologic model for a proposed Edmonton landfill site. Canadian Geothechnical Journal, 40, 551-558.

Pérez-Bielsa, C., Lambán, L.J., Plata, J.L., Rubio, F.M. y Soto, R. 2012. Characterization of a karstic aquifer using magnetic resonance sounding (MRS) and electrical resistivity tomography (ERT): A case-study of Estaña Lakes (Northern Spain). Hydrogeology Journal, 20, 1045-1059.

Pérez-Bielsa, C. 2013. Funcionamiento hidrogeológico de un humedal hipogénico de origen kárstico en las sierras marginales pirenaicas. Tesis doctoral, Universidad Complutense de Madrid, 335 pp. 
Plata, J.L., Rubio, F.M., Coronel, J., Rey, C. and Arias, M., 2007. Revisión de la información geofísica existente en el acuífero Almonte-Marismas (Doñana). Informe inédito. Centro de Documentación del IGME, Madrid. Tomo $1 \mathrm{~N}^{\circ}$ F.D. 63755,276 págs and Tomo $2 \mathrm{~N}^{\circ}$ F.D. 63756, 283 págs.

Plata, J.L. y Rubio, F.M. 2009. Informe de las actividades geofísicas efectuadas en 2008 en las lagunas de Estaña (Huesca). Informe dentro del proyecto: Funcionamiento hidrogeológico de humedales relacionados con las aguas subterráneas en la Cuenca del Ebro. Informe inédito. Centro de Documentación del IGME, Madrid. $N^{\circ}$ F.D. 63764, 200 págs.

Plata, J.L., Rubio, F.M. y Perez-Bielsa, C. 2010. Interpretación integrada de sondeos de resonancia magnética, perfiles de resistividad y testificación de sondeos en las lagunas de Estaña (Huesca). Informe inédito. Centro de Documentación del IGME, Madrid. N F.D. 63939, 226 págs. + anexos

Ratnakumari, Y., Rai, S. N., Thiagarajan, S. y Kumar, D. 2012. 2D Electrical resistivity imaging for delineation of deeper aquifers in a part of the Chandrabhaga river basin, Nagpur District, Maharashtra, India. Current Science, 102 (1), 61-69.

Ruiz, J.M., Rubio, F.M., Ibarra, P., García de Domingo, A., Heredia, J. y Araguas, L. 2006. Contribución de la tomografía eléctrica en la caracterización del sistema hidrogeológico de la laguna de Fuente de Piedra (Málaga). Las aguas subterráneas en los países mediterráneos, vol. 1, Serie: HIDROGEOLOGÍA Y AGUAS SUBTERRÁNEAS, 17, IGME, Madrid, 353- 358.

Salazar-Rincón, A., Mata-Campo, P., Rico-Herrero, M.T., Valero-Garcés, B.L., Oliva-Urcia, B., Ibarra, P., Rubio, F.M. y Grupo Horda. 2013. El paleolago de La Larri (valle de Pineta, Pirineos): significado en el contexto del último máximo glaciar en el Pirineo. Cuadernos de Investigación Geográfica, 39 (1), 97-116.

Zhou, W., Beck, B.F. and Adams, A.L. 2002. Effective electrode array in mapping karst hazards in electrical resistivity tomography. Environmental Geology, 42, 922-928.

Recibido: octubre 2015

Revisado: enero 2016

Aceptado: octubre 2016

Publicado: marzo 2017 